TITLE:

\title{
Plastic flow in two-dimensional solids
}

$\operatorname{AUTHOR}(S)$ :

Onuki, A

\section{CITATION:}

Onuki, A. Plastic flow in two-dimensional solids. Physical Review E 2003, 68(6): 061502.

ISSUE DATE:

2003-12

URL:

http://hdl.handle.net/2433/49912

RIGHT:

Copyright 2003 American Physical Society 
PHYSICAL REVIEW E 68, 061502 (2003)

\title{
Plastic flow in two-dimensional solids
}

\author{
Akira Onuki \\ Department of Physics, Kyoto University, Kyoto 606-8502, Japan
}

(Received 8 April 2003; published 11 December 2003)

\begin{abstract}
A time-dependent Ginzburg-Landau model of plastic deformation in two-dimensional solids is presented. The fundamental dynamic variables are the displacement field $\boldsymbol{u}$ and the lattice velocity $\boldsymbol{v}=\partial \boldsymbol{u} / \partial t$. Damping is assumed to arise from the shear viscosity in the momentum equation. The elastic energy density is a periodic function of the shear and tetragonal strains, which enables the formation of slips at large strains. In this work we neglect defects such as vacancies, interstitials, or grain boundaries. The simplest slip consists of two edge dislocations with opposite Burgers vectors. The formation energy of a slip is minimized if its orientation is parallel or perpendicular to the flow in simple shear deformation and if it makes angles of $\pm \pi / 4$ with respect to the stretched direction in uniaxial stretching. High-density dislocations produced in plastic flow do not disappear even if the flow is stopped. Thus large applied strains give rise to structurally disordered states, which are metastable due to the Peierls potential. We divide the elastic energy into an elastic part due to affine deformation and a defect part. The latter represents degree of disorder and is nearly constant in plastic flow under cyclic straining.
\end{abstract}

DOI: 10.1103/PhysRevE.68.061502

PACS number(s): 83.50.-v, 62.20.Fe, 61.72.Lk, 81.40.Lm

\section{INTRODUCTION}

Plastic flow has long been studied in crystalline and amorphous solids and in glassy polymers. In crystals irreversible motions of dislocations give rise to plastic deformation and large strains produce high-density dislocations [1,2]. The nonlinear flow properties are very sensitive to the amount of such defects and strongly dependent on the deformation history. Simulations of dynamics of dislocation lines have recently been performed but are still most difficult [3-6].

In amorphous solids at low temperature $T$ [7-13], salient features are as follows.

(i) Shear strains tend to be localized in narrow shear bands in plastic flow above a yield stress. The width of such shear bands is microscopic in the initial stage [14] but can grow to mesoscopic sizes [15], sometimes resulting in fracture. Shear bands were numerically realized at large shear strains in molecular dynamics (MD) simulations of twodimensional (2D) two-component glasses [16,17] and in simulations of a 2D phenomenological stochastic model [18].

(ii) As another aspect, in 3D MD simulations on model two-component glasses at low $T$, Takeuchi et al. [19] observed heterogeneities among mobile and immobile regions after application of shear strains. In 2D and 3D MD simulations on model supercooled binary mixtures above $T_{\mathrm{g}}$, similar or much more extended dynamic heterogeneities have been detected in quiescent states [20-23] and found to be sensitively suppressed by applied shear flow [24].

(iii) Furthermore, at rather high $T\left(\gtrsim 0.7-0.8 T_{\mathrm{g}}\right.$ ) (where $T_{\mathrm{g}}$ is the glass transition temperature), shear deformation occurs quasihomogeneously (still involving many particles in each configuration change), leading to highly viscous nonNewtonian behavior [24-27].

(iv) We also mention a phenomenological approach to reproduce shear bands from constitutive equations for the elastic and plastic deformations [28].

Glassy polymers also behave analogously [13,29,30], where shear bands appear above a yield stress at low $T$ [31], and highly viscous non-Newtonian flow and significant elongation of the chain shapes occur at elevated $T[25,32]$. In glassy polymers, the entropic stress arising from molecular orientations becomes significant at large strains and such systems behave like cross-linked rubbers [13,29,30].

Recently much attention has been paid to jamming rheology observed in sheared states of supercooled liquids, soft glassy materials such as dense microemulsions, or granular materials [33]. In these systems, the thermal agitation effect is very small if the particle size is large, but universal constrained dynamics is realized under external forces. In supercooled liquids (at relatively high $T$ ) [24] and dense microemulsions (at effectively low $T$ ) [34,35], mesoscopic dynamic heterogeneity and strong shear-thinning behavior have been observed, but shear bands have not been identified. In granular materials (at effectively zero temperature), strain localization is most conspicuous [36-38].

In our recent work [39] we constructed a 2D nonlinear strain theory taking into account the underlying local periodic lattice structure, where the elastic energy density is periodic with respect to the shear and tetragonal strains. There, we found that plastic flow starts with appearance of slips. In this work we will show that the simplest slips consist of two edge dislocations having opposite Burgers vectors with size $a$ ( $a$ being the lattice constant) and they grow into mesoscopic shear bands as the applied strain is increased. Under uniaxial stretching [40], well developed shear bands were already numerically realized $[17,18]$ and will be realized also in our simulations. These shear bands make angles of $\pm \pi / 4$ with respect to the stretched direction in agreement with observations in various amorphous materials [8-11,29] including granular materials $[36,37]$. These angles will be shown to minimize the elastic energy of incipient slips in this paper.

In crystalline solids, dislocation pairs in 2D or dislocation loops in 3D forming slip lines or surfaces should be nucleated at the inception of plastic deformation (in addition to preexisting dislocations). In amorphous solids, it has been 
controversial whether dislocations themselves can be well defined or not $[7,10]$. It is not obvious how to characterize the local rearrangement processes as to their shapes and sizes. In MD simulations on binary mixtures, they have been detected as clusterlike objects with various visualization methods [19-25,41]. If the size ratio of the constituent two species is chosen such that crystallization is most suppressed, strong frustration occurs in the packing of large and small particles in jammed states. Then the local crystalline order can be well defined only over short distances. However, in a 2D amorphous soap bubble raft, Argon and Kuo [42] observed that nucleation of a dislocation pair gave rise to a small-scale slip but such dislocations did not glide more than two to three bubble distances. It is worth noting that Deng et al. [16] found extended sliplike strain localization in 2D MD simulations with the size ratio rather close to 1 [see comment (iv) in the last section]. Notice that the displacement field around a slip is localized because the two constituent dislocations have opposite Burgers vectors. As a result, smallscale slips should be well defined even in amorphous solids as long as the slip size does not much exceed the range of the local crystal structure.

The plastic flow phenomena are thus very complex, being influenced by many factors, but they are ubiquitous in various kinds of solidlike materials. The purpose of this paper is to present a well-defined Ginzburg-Landau model consistently taking account of nonlinear elasticity. A merit of this approach is that we can put emphasis on any aspect of the phenomena by controlling the parameters or changing the model itself. We will examine (i) the fundamental flow units, slips, in detail numerically and analytically and (ii) plastic flow numerically in simple shear and elongational (stretching) deformation. To make this paper simplest, as it is the first detailed exposition of our scheme, we will neglect (i) vacancies and interstitials, or a variable $m$ representing the local free volume. A dynamic model including such an additional degree of freedom has already been presented in our previous work [39]. We will also neglect (ii) the configurational frustration effect induced by the size difference between the two species. Introducing these two ingredients will constitute future development of our scheme.

This paper is organized as follows. In Sec. II we will present our dynamic model and explain our numerical method. In Sec. III we will discuss the simplest form of slips numerically obtained from our nonlinear elasticity theory. We will also derive some analytic expressions for the slip formation energy under general strain field starting with the Peach-Koehler theory [43] and compare them with numerical results. In our scheme stationary slip solutions exist for small externally applied strains, where the force balance is achieved in the presence of the Peierls potential energy for the dislocation position in crystals $[44,45]$. Section IV will present numerical results of the stress-strain relations and the patterns of the strains, the elastic energy density, and the displacement vector under applied strains. We will also give a method for dividing the elastic energy into an elastic part due to affine deformation and a defect part.

\section{MODEL EQUATIONS}

\section{A. Elastic energy}

Recently Doi et al. [46] described plastic flow in a highly viscous 2D crystal phase of block copolymers assuming 1D sliding motions. Their theory is analogous to a FrenkelKontorova model for commensurate-incommensurate transitions [47]. Hereafter we present a model to describe plastic flow in 2D [39]. In terms of the displacement vector $\boldsymbol{u}$ $=\left(u_{x}, u_{y}\right)$ from a reference crystal state, we define the strain components as

$$
\begin{aligned}
& e_{1}=\nabla_{x} u_{x}+\nabla_{y} u_{y}, \\
& e_{2}=\nabla_{x} u_{x}-\nabla_{y} u_{y}, \\
& e_{3}=\nabla_{x} u_{y}+\nabla_{y} u_{x},
\end{aligned}
$$

where $\nabla_{x}=\partial / \partial x$ and $\nabla_{y}=\partial / \partial y$. We call $e_{1}$ the dilation strain, $e_{2}$ the tetragonal strain, and $e_{3}$ the shear strain. If we suppose a 2D triangular lattice with lattice constant $a$, the elastic energy should be invariant with respect to the rotations of the reference frame by $\pm n \pi / 3(n=1,2, \ldots)$. Due to this symmetry, the elasticity must be isotropic in the harmonic approximation [48], being characterized by the bulk and shear moduli, $K_{0}$ and $\mu_{0}$, but it depends on the orientational angle $\theta$ of one of the crystal axes with respect to the $x$ axis for large shear strains. Under rotation of the reference frame by $\theta$, the shear strains $e_{2}$ and $e_{3}$ are changed to $e_{2}^{\prime}$ and $e_{3}^{\prime}$, where [48]

$$
\begin{aligned}
& e_{2}^{\prime}=e_{2} \cos 2 \theta+e_{3} \sin 2 \theta, \\
& e_{3}^{\prime}=e_{3} \cos 2 \theta-e_{2} \sin 2 \theta .
\end{aligned}
$$

These relations are obtained from the orthogonal transformations, $\boldsymbol{r}^{\prime}=\overleftrightarrow{U} \cdot \boldsymbol{r}$ and $\boldsymbol{u}^{\prime}=\overleftrightarrow{U} \cdot \boldsymbol{u}$, where $\boldsymbol{r}^{\prime}=\left(x^{\prime}, y^{\prime}\right)$ and $\boldsymbol{u}^{\prime}$ $=\left(u_{x}^{\prime}, u_{y}^{\prime}\right)$ represent the position and the displacement, respectively, in the new reference frame, and $\overleftrightarrow{U}=\left\{U_{i j}\right\}$ with $U_{x x}=U_{y y}=\cos \theta$ and $U_{x y}=-U_{y x}=\sin \theta$. The $e_{2}^{\prime}$ and $e_{3}^{\prime}$ are the tetragonal and shear strains, respectively, in the new reference frame where the $x^{\prime}$ axis is along one of the crystal axes.

The elastic energy is written as $F_{\mathrm{el}}=\int d \boldsymbol{r} f_{\mathrm{el}}$ with the elastic energy density in the form

$$
f_{\mathrm{el}}=\frac{1}{2} K_{0} e_{1}^{2}+\mu_{0} \Phi\left(e_{3}^{\prime}, e_{2}^{\prime}\right),
$$

which is independent of the rotation strain,

$$
\omega=\nabla_{x} u_{y}-\nabla_{y} u_{x}
$$

Note that $e_{1}$ and $\omega$ are invariant with respect to the rotation of the reference frame. The simplest form of $\Phi$ is given by

$$
\begin{aligned}
\Phi\left(e_{3}^{\prime}, e_{2}^{\prime}\right)= & \frac{1}{6 \pi^{2}}\left[3-\cos \pi\left(\sqrt{3} e_{3}^{\prime}-e_{2}^{\prime}\right)\right. \\
& \left.-\cos \pi\left(\sqrt{3} e_{3}^{\prime}+e_{2}^{\prime}\right)-\cos \left(2 \pi e_{2}^{\prime}\right)\right] .
\end{aligned}
$$




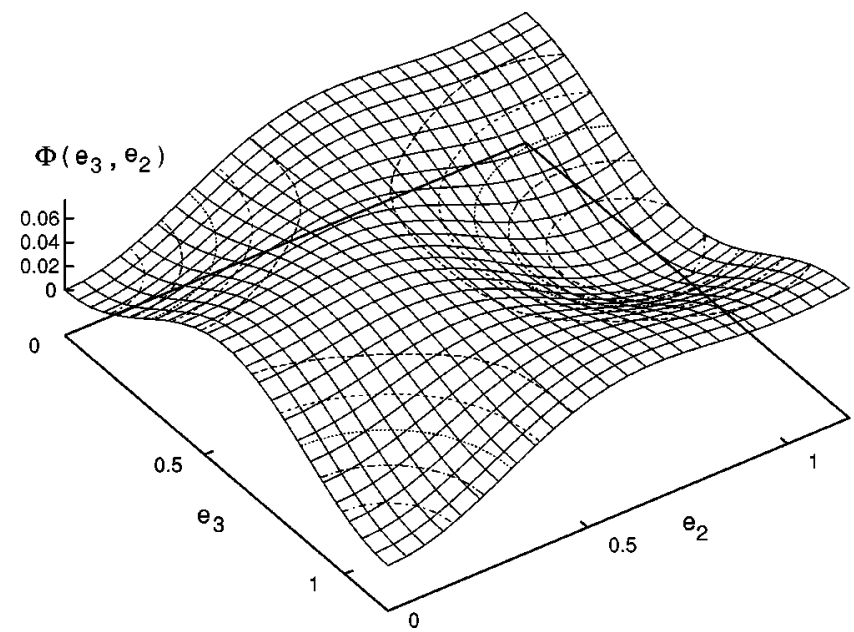

FIG. 1. The scaling function $\Phi\left(e_{3}, e_{2}\right)$ in Eq. (2.5), which is the shear deformation energy density divided by $\mu_{0}$ with one of the crystal axes being along the $x$ axis.

This function is invariant with respect to the rotation $\theta \rightarrow \theta$ $+\pi / 6$, is a periodic function of $e_{3}^{\prime}$ with period $2 / \sqrt{3}$ for $e_{2}^{\prime}$ $=0$ (simple shear deformation), and becomes $\left(e_{2}^{2}+e_{3}^{2}\right) / 2$ for small strains. In Fig. 1 we display $\Phi\left(e_{3}, e_{2}\right)$ supposing $\theta$ $=0$, where one of the crystal axes is along the $x$ axis. We can see a hexagonal lattice structure in the $e_{3}-e_{2}$ plane. As a characteristic feature, Fig. 2 shows that it is almost isotropic or is a function of $e=\left(e_{3}^{2}+e_{2}^{2}\right)^{1 / 2}$ only even for rather large $e(\lesssim 0.5)$ (in the unit cell at the origin). In fact, we have the Taylor expansion,

$$
\begin{aligned}
\Phi\left(e_{3}, e_{2}\right)= & \frac{1}{2} e^{2}-\frac{\pi^{2}}{8} e^{4}+\frac{\pi^{4}}{72} e^{6}+\frac{\pi^{4}}{720}\left(e_{2}^{2}-e_{3}^{2}\right) \\
& \times\left(e^{4}-16 e_{2}^{2} e_{3}^{2}\right)+O\left(e^{8}\right)
\end{aligned}
$$

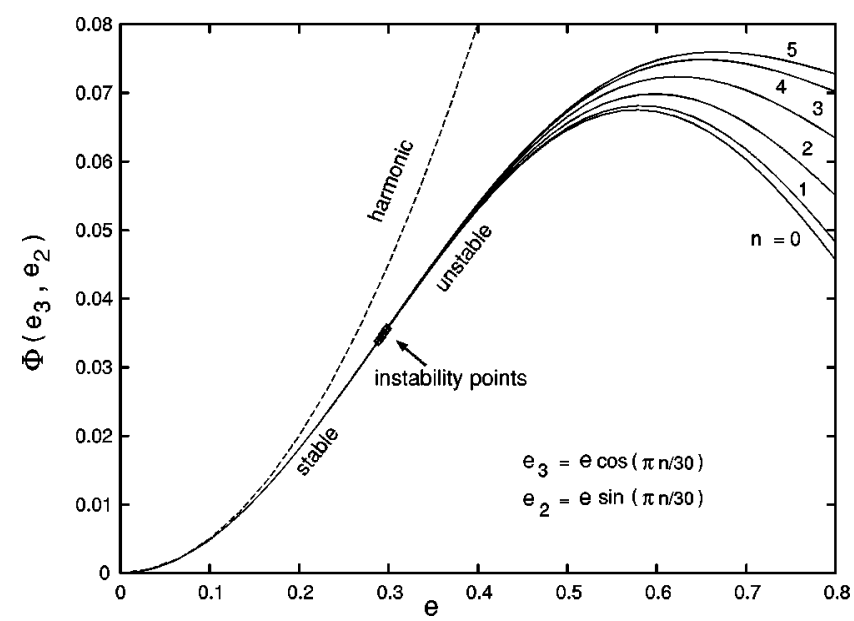

FIG. 2. $\Phi\left(e_{3}, e_{2}\right)$ for various directions in the $e_{3}-e_{2}$ plane. It demonstrates isotropic behavior for $e=\left(e_{3}^{2}+e_{2}^{2}\right)^{1 / 2}<0.5$. The curve for uniaxial stretching $\left(e_{2}=e\right.$ and $\left.e_{3}=0\right)$ coincides with the curve of $n=5$ in the figure. The instability points are marked, which separate stable and unstable regions.

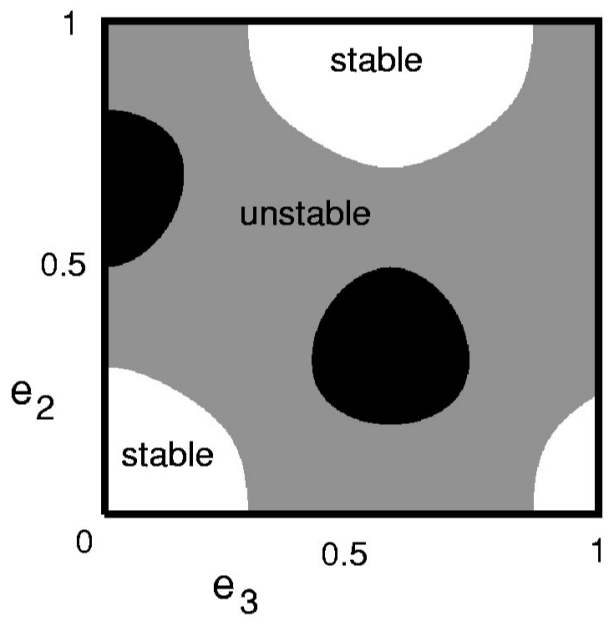

FIG. 3. The stable regions of our elastic energy are shown in white, where the two eigenvalues of $\left\{\Phi_{\alpha \beta}\right\}$ are both positive. In the gray region one of them is negative, and in the black regions both of them are negative.

If we set $e_{3}=e \cos \chi$ and $e_{2}=e \sin \chi$, the fourth term is rewritten as $-\left(\pi^{4} / 720\right) e^{6} \cos (6 \chi)$ and is known to keep the invariance $\chi \rightarrow \chi+\pi / 3$. Anisotropy appears in the terms of order $e^{6}$, but the anisotropic fourth term is at most $10 \%$ of the isotropic third term.

We examine elastic stability of homogeneously strained states. That is, we superimpose infinitesimal strains, $\delta e_{3}$ and $\delta e_{2}$, on $e_{3}$ and $e_{2}$ assumed to be homogeneous. The second order terms in $\Phi\left(e_{3}+\delta e_{3}, e_{2}+\delta e_{2}\right)$ read

$$
\delta^{(2)} \Phi=\frac{\Phi_{33}}{2}\left(\delta e_{3}\right)^{2}+\Phi_{23} \delta e_{3} \delta e_{2}+\frac{\Phi_{22}}{2}\left(\delta e_{2}\right)^{2},
$$

where $\Phi_{\alpha \beta}=\partial^{2} \Phi / \partial e_{\alpha} \partial e_{\beta}(\alpha, \beta=2,3)$. In the stable regions the above second order contribution should be positive definite or the two eigenvalues, $\lambda_{1}$ and $\lambda_{2}$, of the $2 \times 2$ matrix $\left\{\Phi_{\alpha \beta}\right\}$ should be both positive. In the Appendix we will derive this linear stability criterion by solving the linearized version of our dynamic model to be presented in the following section. Figure 3 shows that the system is stable for $e$ $\lesssim 0.3$, where $\Phi$ depends almost only on $e$. This result readily follows if we neglect the anisotropy in $\Phi$ by setting $\Phi\left(e_{2}, e_{3}\right) \cong G\left(e^{2}\right)$. Then some calculations yield

$$
\Phi_{\alpha \beta} \cong 2 G^{\prime} \delta_{\alpha \beta}+4 G^{\prime \prime} e_{\alpha} e_{\beta},
$$

where $G^{\prime}=d G\left(e^{2}\right) / d e^{2}$ and $G^{\prime \prime}=d G^{\prime}\left(e^{2}\right) / d e^{2}$. From Eq. (2.6) we find $G^{\prime} \cong 1 / 2$ and $G^{\prime \prime} \cong-\pi^{2} / 4$. The determinant of $\left\{\Phi_{\alpha \beta}\right\}$ becomes $4 G^{\prime}\left(G^{\prime}+2 G^{\prime \prime} e^{2}\right)$. Thus the stability condition becomes

$$
e<\left(G^{\prime} /\left|2 G^{\prime \prime}\right|\right)^{1 / 2} \cong 0.3
$$

around the origin in the $e_{3}-e_{2}$ plane. The elastic instability with a negative eigenvalue $\left(\lambda_{1}<0\right.$ or $\left.\lambda_{2}<0\right)$ causes rapid relaxation processes resulting in localized slips and stress release. In plastic flow the stability condition is satisfied at most points throughout the system (see Fig. 10). 
If we assume that small solid elements are rotated without shape changes with the local angular velocity $\left(\partial v_{y} / \partial x\right.$ $\left.-\partial v_{x} / \partial y\right) / 2$, the orientation angle $\theta$ is related to the rotation strain as

$$
\theta=\frac{1}{2} \omega+\theta_{0},
$$

where $\theta_{0}$ is the initial value independent of time $t$. This relation was assumed in our previous paper [39]. However, it becomes not well defined when the three crystal axes are rotated differently. For example, let a crystal with the three axes at $\theta=0, \pi / 3$, and $-\pi / 3$ be affinely deformed by a simple shear deformation given by $u_{x}=\gamma y$ and $u_{y}=0\left(e_{3}\right.$ $=\gamma$ and $\left.e_{2}=0\right)$. Then the first axis along the $x$ direction is not rotated, while the other axes are rotated by

$$
\delta \theta_{ \pm}= \pm\left[\tan ^{-1}\left(\frac{\sqrt{3}}{1 \pm \sqrt{3} \gamma}\right)-\frac{\pi}{3}\right]
$$

For $\gamma \ll 1$, we have $\delta \theta_{ \pm} \cong-3 \gamma / 4$, so Eq. (2.10) holds for the average of the three rotation angles, $\left(0+\delta \theta_{+}+\delta \theta_{-}\right) / 3$ $=-\gamma / 2=\omega / 2$. Thus there remains ambiguity in Eq. (2.10) particularly at large strains $e \gtrsim 0.5$ [49]. In this paper, in view of the virtual isotropic behavior of our elastic energy for $e$ $\lesssim 0.5$, we will simply set

$$
\theta=0
$$

throughout the system. To check the appropriateness of this assumption, we have also performed simulations with various homogeneous $\theta$ held fixed in the range $0<\theta<\pi / 6$. For $\theta=\pi / 12$, slip lines become slightly curved in plastic flow and have higher elastic energies (by a few ten \%), but there arises no qualitative change in the patterns and stress-strain relations (see also the comment at the end of Sec. IV).

\section{B. Dynamic equations}

The elastic stress tensor should be defined for general strains. Let us change an arbitrary displacement $\boldsymbol{u}$ by an infinitesimal amount $\delta \boldsymbol{u}$ as $\boldsymbol{u} \longrightarrow \boldsymbol{u}+\delta \boldsymbol{u}$. The incremental change of the elastic energy density is written as [48]

$$
\delta f_{\mathrm{el}}=\sum_{i j} \sigma_{i j} \frac{\partial}{\partial x_{j}} \delta u_{i}
$$

This is just the definition of the elastic stress tensor $\sigma_{i j}$. Under Eq. (2.12) [49] it is symmetric and its components are expressed as

$$
\begin{gathered}
\sigma_{x x}=K_{0} e_{1}+\mu_{0} A_{2}, \quad \sigma_{y y}=K_{0} e_{1}-\mu_{0} A_{2}, \\
\sigma_{x y}=\sigma_{y x}=\mu_{0} A_{3},
\end{gathered}
$$

where $A_{\alpha}=\partial \Phi\left(e_{3}, e_{2}\right) / \partial e_{\alpha}$ are written as

$$
\begin{gathered}
A_{2}=\frac{1}{3 \pi}\left[\cos \left(\sqrt{3} \pi e_{3}\right) \sin \left(\pi e_{2}\right)+\sin \left(2 \pi e_{2}\right)\right], \\
A_{3}=\frac{1}{\sqrt{3} \pi} \sin \left(\sqrt{3} \pi e_{3}\right) \cos \left(\pi e_{2}\right) .
\end{gathered}
$$

For small strains we have $A_{2} \cong e_{2}$ and $A_{3} \cong e_{3}$ to reproduce the isotropic, linear elastic theory.

We assume that the lattice velocity

$$
\boldsymbol{v}=\frac{\partial}{\partial t} \boldsymbol{u}
$$

obeys

$$
\rho \frac{\partial}{\partial t} \boldsymbol{v}=\boldsymbol{\nabla} \cdot \stackrel{\leftrightarrow}{\sigma}+\eta_{0} \nabla^{2} v+\nabla \cdot \overleftrightarrow{\sigma}_{\mathrm{R}}
$$

where we introduce the shear viscosity $\eta_{0}$ but neglect the bulk viscosity $[50,51]$. The $\stackrel{\leftrightarrow}{\mathrm{R}}_{\mathrm{R}}=\left\{\sigma_{i j}^{\mathrm{R}}\right\}$ is a symmetric random stress tensor and satisfies $\sigma_{x x}^{\mathrm{R}}+\sigma_{y y}^{\mathrm{R}}=0$, because the bulk viscosity is neglected, and is related to $\eta_{0}$ by [48]

$$
\left\langle\sigma_{i j}^{\mathrm{R}}(\boldsymbol{r}, t) \sigma_{i j}^{\mathrm{R}}\left(\boldsymbol{r}^{\prime}, t^{\prime}\right)\right\rangle=2 k_{\mathrm{B}} T \eta_{0} \delta\left(\boldsymbol{r}-\boldsymbol{r}^{\prime}\right) \delta\left(t-t^{\prime}\right) .
$$

The mass density $\rho$ will be treated as a constant in Eq. (2.17). This is justified when the deviation $\delta \rho=\rho-\langle\rho\rangle$ is assumed to be much smaller than the average $\langle\rho\rangle$ [52]. In the usual linear elasticity theory [50] it is related to the dilation strain $e_{1}$ as $\delta \rho \cong-\rho e_{1}$, so we are assuming $\left|e_{1}\right| \ll 1$ (and coincidence of the lattice and mass velocities [39]). In our simulation in the plastic flow regime at $\dot{\gamma}=10^{-3}$, for example, $\left|e_{1}\right|$ attains a maximum in a range of $0.2-0.3$ and the variance $\sqrt{\left\langle e_{1}^{2}\right\rangle}$ increases up to about 0.06 .

Due to the presence of the random stress, $\boldsymbol{u}$ and $\boldsymbol{v}$ are random variables and Eqs. (2.16) and (2.17) constitute nonlinear Langevin equations [48]. Their equilibrium distribution attained in the unstrained condition is given by $P$ $\propto \exp \left(-F / k_{\mathrm{B}} T\right)$, where the total free energy $F$ is the sum of the elastic energy $F_{\mathrm{el}}$ and the kinetic energy as

$$
F=\int d \boldsymbol{r}\left(f_{\mathrm{el}}+\frac{\rho}{2} \boldsymbol{v}^{2}\right) .
$$

Furthermore, if the random stress is omitted in Eq. (2.17) and the dynamic equations are treated as deterministic ones, the time derivative of $F$ is non-negative definite in the unstrained condition as

$$
\frac{d}{d t} F=-\int d \boldsymbol{r} \sum_{i j} \eta_{0}\left(\nabla_{i} v_{j}\right)^{2} \leqslant 0
$$

where use is made of the relation,

$$
\nabla \cdot \stackrel{\leftrightarrow}{\sigma}=-\frac{\delta}{\delta \boldsymbol{u}} F
$$


The stationary condition $d F / d t=0$ is attained for $\boldsymbol{v}=\mathbf{0}$ and $\nabla \cdot \overleftrightarrow{\sigma}=\mathbf{0}$. This is a condition to guarantee self-consistency of the dynamic equations which have stable equilibrium solutions.

In the Appendix we will examine the linearized dynamic equations of our model around homogeneously strained states to obtain two sound modes in the stable region.

\section{Dimensionless forms}

We make our equations dimensionless measuring space in units of the lattice constant $a$ and time in units of

$$
\tau_{0}=\left(\rho / \mu_{0}\right)^{1 / 2} a .
$$

The stress components and the elastic energy density are measured in units of $\mu_{0}$ and the elastic energy in units of $\mu_{0} a^{2}$, while the strains remain unscaled. To avoid introducing too many symbols, we will rewrite the scaled position vector $a^{-1} \boldsymbol{r}$, time $\tau_{0}^{-1} t$, displacement vector $a^{-1} \boldsymbol{u}$, and velocity $\tau_{0} a^{-1} \boldsymbol{v}$ simply as $\boldsymbol{r}, t, \boldsymbol{u}$, and $\boldsymbol{v}$ using the original notation. Then, in the dimensionless $\overleftrightarrow{\sigma}, \mu_{0}$ is replaced by 1 and $K_{0}$ by the ratio

$$
\lambda=K_{0} / \mu_{0} .
$$

In terms of the scaled quantities the equilibrium distribution is written as

$$
P \propto \exp \left[-\frac{1}{\epsilon_{\mathrm{th}}} \int d \boldsymbol{r}\left(\frac{\lambda}{2} e_{1}^{2}+\Phi\left(e_{3}, e_{2}\right)+\frac{1}{2} \boldsymbol{v}^{2}\right)\right],
$$

where

$$
\epsilon_{\mathrm{th}}=k_{\mathrm{B}} T / \mu_{0} a^{2} .
$$

The parameter $\epsilon_{\text {th }}$ represents the degree of the thermal fluctuations (being proportional to $T$ ) and is an important parameter, for example, in describing the decay of metastable states by thermal agitations. If the dynamic equation (2.17) is made dimensionless, the dimensionless viscosity is given by

$$
\eta_{0}^{*}=\eta_{0} a^{-1}\left(\rho \mu_{0}\right)^{-1 / 2} .
$$

In Eq. (2.18) the noise strength $k_{\mathrm{B}} T \eta_{0}$ is replaced by $\epsilon_{\mathrm{th}} \eta_{0}^{*}$.

\section{Numerical method}

We integrate Eqs. (2.16) and (2.17) in the dimensionless units on a $128 \times 128$ square lattice represented by $(n, m)$ $(1 \leqslant n, m \leqslant 128)$ with $x=n \Delta x$ and $y=m \Delta x$. The modulus ratio $\lambda$ in Eq. (2.23) is set equal to 1. For simplicity, the mesh size $\Delta x$ and the dimensionless viscosity in Eq. (2.26) are set equal to 1 :

$$
\Delta x=1, \quad \eta_{0}^{*}=1 .
$$

The first relation means that the mesh size is just equal to the lattice constant, and the second one is rewritten as $\eta_{0}$ $=a\left(\rho \mu_{0}\right)^{1 / 2}$.

We next explain our boundary conditions employed. At the bottom $y=0$, we always set $u_{x}=u_{y}=0$. At the top $y$
$=L_{0}=128$, we set $u_{x}=\gamma L_{0}$ and $u_{y}=0$ in the presence of applied shear strain $\gamma$, while $u_{x}=-u_{y}=\epsilon L_{0} / 2$ in the presence of applied tetragonal strain $\epsilon$. In the $x$ direction, we impose the periodic boundary condition, $\boldsymbol{u}\left(x+L_{0}, y, t\right)$ $=\boldsymbol{u}(x, y, t)$.

We are interested in slips across which the atomic displacement is discontinuous by the lattice constant $a$. In this paper, by setting $\Delta x=a$, we try to realize such singular objects numerically. For this purpose it is convenient to define the strains and tensors on the middle points $(n+1 / 2, m$ $+1 / 2$ ), while the vectors are defined at the lattice points $(n, m)$. For a vector component $A$ (say, $A=u_{x}$ ), $\nabla_{x} A$ and $\nabla_{y} A$ at $(n+1 / 2, m+1 / 2)$ are defined as $[A(n+1, m+1)$ $-A(n, m)+A(n+1, m)-A(n, m+1)] / 2$ and $[A(n+1, m$ $+1)-A(n, m)-A(n+1, m)+A(n, m+1)] / 2$, respectively, using $A$ at the four points $(n+1 / 2 \pm 1 / 2, m+1 / 2 \pm 1 / 2)$. In the same manner, we may construct the vector $\boldsymbol{\nabla} \cdot \overleftrightarrow{\sigma}$ at $(n, m)$ using the stress components at the four points $(n \pm 1 / 2, m$ $\pm 1 / 2$ ). With this space discretization, slips consisting of a straight line segment become well defined if their angle with respect to the $x$ or $y$ axis is 0 or $\pi / 4$. For other slip orientations, zigzag points appear along the slip line segment and an extra elastic energy is needed. On the other hand, if we define all the quantities on $(n, m)$, slip discontinuity takes place over a few lattice sizes, but macroscopic features such as the stress-strain relation remain almost unchanged. Furthermore, we will suppose simple shear or uniaxial deformation and, as will be shown in the following section, the preferred slip orientation angle is 0 or $\pi / 4$ with respect to the $x$ or $y$ axis. By this reason our simple numerical scheme seems to be allowable at least in this first attempt.

\section{SLIPS}

In our model system fundamental flow units in plastic deformation are slips composed of a pair of edge dislocations with opposite Burger vectors (dislocation dipoles). They are analogous to quantum vortex rings in superfluid helium [48]. Their elastic structure far from the dislocation cores may well be described by the linear elasticity theory, but nonlinear elasticity theory is needed (i) to suppress the divergence of the stress at the cores and (ii) to stabilize the slips themselves when they adjust to the crystal structure. Slips are not in a stationary state in the linear elasticity theory in the absence of impurities, etc., which can trap dislocations, as will be evident in Eq. (3.20). In our nonlinear theory, those along the $x$ axis $(\theta=0)$ can be in a stationary metastable state if their length is a multiple of the lattice constant. This is consistent with the Peierls-Nabarro theory $[44,45]$, which takes into account the discreteness of the crystal structure and gives a periodic Peierls potential energy for the position of the dislocation center.

\section{A. Slips in linear elasticity theory}

To begin with, let us write the solution of an edge dislocation as $\boldsymbol{u}=b \boldsymbol{u}^{\mathrm{Le}}=\left(b u_{x}^{\mathrm{Le}}, b u_{y}^{\mathrm{Le}}\right)$, whose Burgers vector is assumed to be along the $x$ direction and is written as $\boldsymbol{b}$ $=(b, 0)$. The linear elasticity theory $[50]$ gives 


$$
\begin{gathered}
u_{x}^{\mathrm{Le}}=\frac{1}{2 \pi}\left[\tan ^{-1}\left(\frac{y}{x}\right)+\frac{1}{2(1-\nu)} \frac{x y}{x^{2}+y^{2}}\right], \\
u_{y}^{\mathrm{Le}}=\frac{-1}{4 \pi(1-\nu)}\left[(1-2 \nu) \ln \sqrt{x^{2}+y^{2}}+\frac{x^{2}}{x^{2}+y^{2}}\right] .
\end{gathered}
$$

In the 3D theory the dislocation line is along the $z$ axis and $\nu$ is $3 \mathrm{D}$ Poisson's ratio. In our $2 \mathrm{D}$ theory $\nu$ is related to $\lambda$ in Eq. (2.23) as

$$
\nu=\frac{1}{2}-\frac{1}{2 \lambda} .
$$

In our simulations we set $\lambda=1$ and hence $\nu=0$. In the linear theory a slip is a superposition of two edge dislocations with opposite Burgers vectors expressed as

$$
\boldsymbol{u}^{\mathrm{Ls} \pm}= \pm\left[\boldsymbol{u}^{\mathrm{Le}}\left(x-\frac{\ell}{2}, y\right)-\boldsymbol{u}^{\mathrm{Le}}\left(x+\frac{\ell}{2}, y\right)\right]
$$

where $\ell$ is the slip length and $b= \pm 1$ (both in units of $a$ ). The slip line segment is between the two points $(-\ell / 2,0)$ and $(\ell / 2,0)$. The + sign corresponds to a clockwise slip (type $C$ ), and the - sign to a counterclockwise slip (type CC). The displacement vector around a slip is clockwise (counterclockwise) for type $C$ (type CC) (as will be evident in Fig. 5 below). Across the line segment $|x|<\ell / 2$ and $y$ $=0$, the displacement is discontinuous as

$$
u_{x}^{\mathrm{Ls} \pm}(x, y+0)-u_{x}^{\mathrm{Ls} \pm}(x, y-0)= \pm 1 .
$$

The corresponding strains are written as

$$
\begin{gathered}
e_{1}^{\mathrm{Ls} \pm}=\frac{ \pm(2 \nu-1)}{2 \pi(1-\nu)}\left[\frac{y}{x_{+}^{2}+y^{2}}-\frac{y}{x_{-}^{2}+y^{2}}\right], \\
e_{2}^{\mathrm{Ls} \pm}=\frac{\mp 1}{\pi(1-\nu)}\left[\frac{x_{+}^{2} y}{\left(x_{+}^{2}+y^{2}\right)^{2}}-\frac{x_{-}^{2} y}{\left(x_{-}^{2}+y^{2}\right)^{2}}\right], \\
e_{3}^{\mathrm{Ls} \pm}=\frac{ \pm 1}{2 \pi(1-\nu)}\left[\frac{x_{+}\left(x_{+}^{2}-y^{2}\right)}{\left(x_{+}^{2}+y^{2}\right)^{2}}-\frac{x_{-}\left(x_{-}^{2}-y^{2}\right)}{\left(x_{-}^{2}+y^{2}\right)^{2}}\right] \\
\pm \delta(y) \Theta\left(\ell^{2} / 4-x^{2}\right),
\end{gathered}
$$

where $x_{ \pm}=x \mp \ell / 2$, and $\Theta(\zeta)$ is the step function being equal to 1 for $\zeta>0$ and to 0 for $\zeta<0$. There appears no $\delta$ function in the dilation and tetragonal strains. The strains diverge at the cores where the linear elasticity theory breaks down.

\section{B. Slips in nonlinear elasticity theory}

Next we numerically construct the corresponding slip solution in our nonlinear elasticity theory for integer $\ell$. By starting with the linear solution $\boldsymbol{u}^{\text {Ls } \pm}$ in Eq. (3.3) at $t=0$ and neglecting the random stress $\overleftrightarrow{\sigma}_{R}$, we integrate Eqs. (2.16) and (2.17) to seek the steady solution $\boldsymbol{u}^{\mathrm{s} \pm}$ attained after transient relaxation. That is,

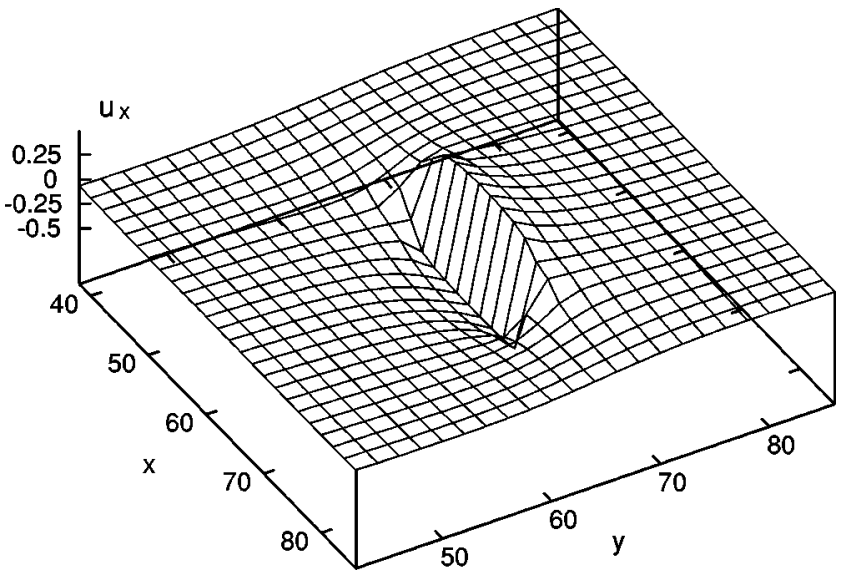

FIG. 4. The $x$ component of the displacement for a numerically calculated type $C$ slip with length 10 along the $x$ axis in the unstrained condition.

$$
\boldsymbol{u}^{\mathrm{s} \pm}(x, y)=\lim _{t \rightarrow \infty} \boldsymbol{u}(x, y, t),
$$

where $\boldsymbol{u}(x, y, 0)=\boldsymbol{u}^{\mathrm{Ls} \pm}(x, y)$. The core points of the linear solution, where the strains diverge, are placed at middle points $(n+1 / 2, m+1 / 2)$ of the mesh of integration at $t=0$. The limit $\boldsymbol{u}^{\mathrm{s} \pm}$ is a steady metastable solution satisfying the mechanical equilibrium condition,

$$
\boldsymbol{\nabla} \cdot \stackrel{\leftrightarrow}{\sigma}=\mathbf{0}
$$

It nearly coincides with the linear solution $\boldsymbol{u}^{\mathrm{Ls} \pm}$ far from the dislocation cores $\left(|x \pm \ell / 2|^{2}+y^{2} \geq 3\right.$ in our case) and keeps satisfying the slip condition (3.4). The strain and stress components calculated from $\boldsymbol{u}^{\mathrm{s} \pm}$ are finite even in the core regions. In fact, in the presence of a slip along the $x$ direction, the maximum values attained by $\left|e_{1}\right|,\left|e_{2}\right|,\left|e_{3}\right|$, and $\left|\sigma_{x y}\right|$ in the core regions are about $0.18,0.08,1.1$, and 0.1 , respectively. In Fig. 4 we show the $x$ component $u_{x}=u_{x}^{\mathrm{s}+}$ of a clockwise slip with $\ell=10$ in the unstrained condition, which is discontinuous by 1 across the slip segment.

The elastic energy of a slip in our nonlinear theory is then of central importance. We will neglect the Peierls potential energy for the time being. We generally assume that a slip line is oblique to the $x$ axis making an angle of $\varphi$ and is under externally applied strains,

$$
\left\langle e_{3}\right\rangle=\gamma, \quad\left\langle e_{2}\right\rangle=\epsilon, \quad\left\langle e_{1}\right\rangle=0
$$

where $\langle\cdots\rangle$ is the space average. The slip energy to create a single slip is defined by

$$
F_{\text {slip }}=F-F_{0}=\int d \boldsymbol{r}\left(f_{\mathrm{el}}-f_{\mathrm{el}}^{0}\right),
$$

where $f_{\mathrm{el}}$ is the elastic energy density in our nonlinear theory with one slip calculated numerically, and $f_{\mathrm{el}}^{0}=\Phi(\gamma, \epsilon)$ is that in a homogeneously strained state. In the unstrained condition $(\gamma=\epsilon=0)$, the expression $F_{\text {slip }}=\ln \ell / 2 \pi(1-\nu)$ (in units of $\left.\mu_{0} a^{2}\right)$ is well known [53]. 
Dislocation motions perpendicular to the slip line (climb motions) create a large number of defects $(\propto \ell)$ and the energy needed is very large, so they may be neglected [54]. On the other hand, dislocation motions along the slip line (glide motions) involve displacements of a relatively small number of particles (of order 10 for a unit-length motion as can be seen in the inset of Fig. 9) and hence play a major role in plastic flow. A force $\mathcal{F}=\left(\mathcal{F}_{x}, \mathcal{F}_{y}\right)$ acting on a dislocation under an applied stress $\left\{\sigma_{i j}^{\mathrm{ex}}\right\}$ may be calculated using the Peach-Koehler theory $[1,2,43]$. For $\boldsymbol{b}=(b, 0)$ along the $x$ axis, the components of the force are written as

$$
\mathcal{F}_{x}=-\sigma_{x y}^{\mathrm{ex}} b, \quad \mathcal{F}_{y}=\sigma_{x x}^{\mathrm{ex}} b .
$$

(i) For a slip along the $x$ axis, $\mathcal{F}_{y}$ is canceled by the force due to an additional elastic deformation in the surrounding medium. If the dislocation on the left is fixed and that on the right is moved by $\delta \ell$ along the $x$ axis, the change of $F_{\text {slip }}$ is equal to $\mathcal{F}_{x} \delta \ell$. Here the Peierls force is neglected. Therefore,

$$
\frac{\partial}{\partial \ell} F_{\mathrm{el}}=\mathcal{F}_{x}=-\sigma_{x y}^{\mathrm{ex}} b
$$

The $\sigma_{x y}^{\mathrm{ex}}$ consists of the stress produced by the dislocation on the left and the externally applied stress. For small $\gamma$ the externally applied stress is $\mu_{0} \gamma$, so in the units in which $\mu_{0}=b=1$ we obtain

$$
F_{\text {slip }}=\frac{\ln \ell}{2 \pi(1-\nu)} \mp \gamma \ell,
$$

where - is for type $C$ and + is for type CC.

(ii) For general angle $\varphi$ of the slip with respect to the $x$ axis, we rotate the reference frame by $\varphi$ to obtain the shear strain $\gamma^{\prime}=\gamma \cos 2 \varphi-\epsilon \sin 2 \varphi$ in the new reference frame from Eq. (2.2). Therefore,

$$
F_{\text {slip }}=\frac{\ln \ell}{2 \pi(1-\nu)} \mp(\gamma \cos 2 \varphi-\epsilon \sin 2 \varphi) \ell .
$$

If $\varphi$ is varied in Eq. (3.13), $F_{\text {slip }}$ is minimized for

$$
\varphi=\frac{1}{2}(n \pi-\alpha) \quad(n=0,1,2, \ldots),
$$

where $n$ is even for type $C$ and odd for type CC, and $\alpha$ is determined by

$$
\cos \alpha=\frac{\gamma}{\sqrt{\gamma^{2}+\epsilon^{2}}}, \quad \sin \alpha=\frac{\epsilon}{\sqrt{\gamma^{2}+\epsilon^{2}}} .
$$

For simple shear deformation with $\gamma>0$ and $\epsilon=0$, the most favorable slip orientation with the lowest $F_{\text {slip }}$ is $\varphi=0$ for type $C$ and $\varphi=\pi / 2$ for type CC. For uniaxial stretching with $\gamma=0$ and $\epsilon>0$, it is given by $\varphi=-\pi / 4$ for type $\mathrm{C}$ and $\varphi$ $=\pi / 4$ for type $\mathrm{CC}$, in agreement with the experiments [8-11,29,36,37].

As will be shown in the appendix, the slip directions determined by Eq. (3.14) are perpendicular to the wave vectors which minimize the angle-dependent sound velocity follow- (a) Shear deformation
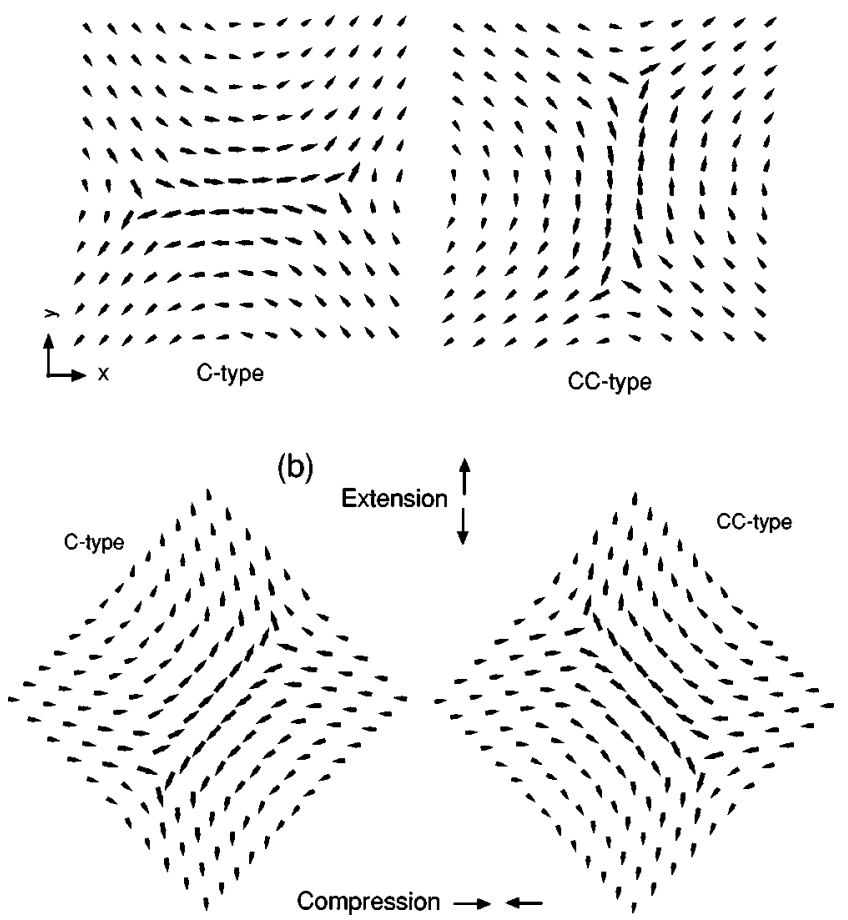

FIG. 5. The displacement vector $\boldsymbol{u}$ around type $C$ and type CC slips with length 10 . The orientations in (a) are most favorable in shear deformation, while those in (b) are most favorable in uniaxial stretching. The arrows are from the original undeformed position to the displaced position.

ing from our linearized dynamic equations, or perpendicular to the softest directions for the sound modes. This coincidence is obtained with the aid of the isotropic behavior (2.8), so it is not a general result for anisotropic solids. We remark that previous theories of strain localization $[37,55,56]$ are based on 1D analysis, where all the quantities vary only in one direction $\boldsymbol{n}$ normal to the plane of the band, and reduce to linear stability analysis for small amplitude perturbations for the wave vector in the direction of $\boldsymbol{n}$.

In Fig. 5 we display the displacement vector $\boldsymbol{u}$ around type $C$ and type CC slips calculated in the unstrained condition. The slips are oriented in the most favorable directions in shear deformation in (a) and in uniaxial stretching in (b). Away from the slips the directions of $\boldsymbol{u}$ continuously change to those of the macroscopic deformation supposed to be applied. In Fig. 6 the slip energy $F_{\text {slip }}$ of type $C$ slips with $\ell$ $=10,20$, and 30 is shown as a function of the applied shear strain $\gamma$. For $|\gamma| \lesssim 0.05$ we confirm Eq. (3.12) with the sign. For larger $\gamma$ the linear relation $\partial F_{\text {slip }} / \partial \gamma=$ const does not hold. For $\gamma<\gamma_{\mathrm{c} 1}(\sim-0.1)$ or for $\gamma>\gamma_{\mathrm{c} 2}(\sim 0.1)$, a steady metastable solution becomes nonexistent and, as a result, the slip grows up to the system length or shrinks to vanish in the simulation. In Fig. 7 the slip energy $f_{\mathrm{el}}-f_{\mathrm{el}}^{0}$ with $f_{\mathrm{el}}^{0}=\Phi(\gamma, 0)$ is displayed for the three values $\gamma=0$, 0.065 , and -0.04 . Interestingly, the elastic energy density in the middle region between the two dislocations at the ends is decreased for positive $\gamma$ and increased for negative $\gamma$, giving rise to the contribution $-\ell \gamma$ in the slip energy. In the core 


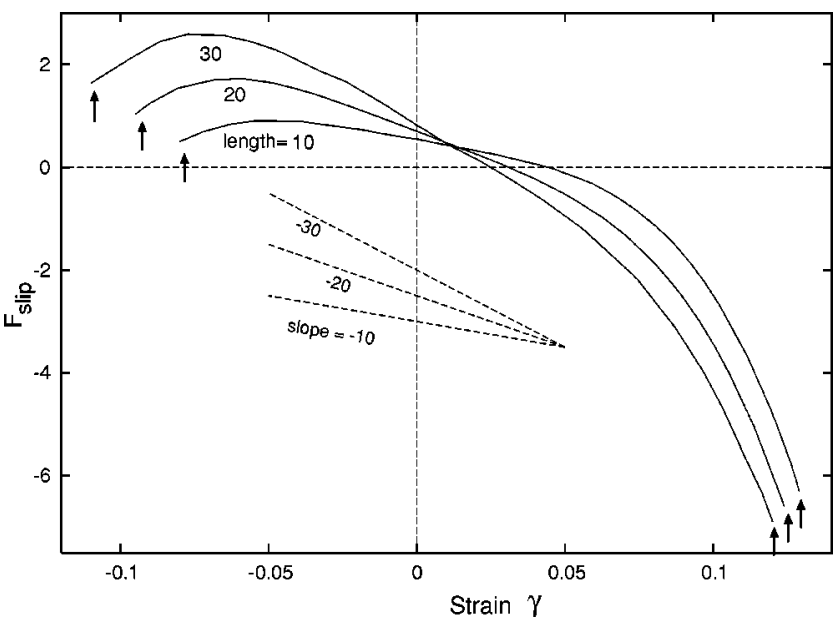

FIG. 6. The slip energy $F_{\text {slip }}$ vs applied shear strain $\gamma$ for type $C$ slips with length $\ell=10,20$, and 30 oriented along the $x$ axis. For $|\gamma|<0.05$ relation (3.12) holds with slope $-\ell$. The arrows indicate the instability points where expansion or shrinking of the slips occurs.

region, $f_{\mathrm{el}}$ has two peaks and is rather insensitive to applied strains in our model.

Furthermore, in Fig. 8 we numerically demonstrate coincidence of $\partial F_{\text {slip }} / \partial \gamma$ and the space integral of $\sigma_{x y}-\gamma$ for a single isolated slip in simple shear deformation. This relation may be obtained from Eq. (2.1). If $\gamma$ is increased by an infinitesimal amount $\delta \gamma$, the change of $F_{\text {slip }}$ is written in terms of the incremental displacement $\delta u_{i}$ as

$$
\delta F_{\text {slip }}=\int d \boldsymbol{r} \sum_{i j}\left[\sigma_{i j}-\sigma_{i j}^{0}\right] \frac{\partial}{\partial x_{j}} \delta u_{i}
$$

where $\left\{\sigma_{i j}^{0}\right\}$ is the stress in the homogeneous state and use is made of the relation for the space average $\left\langle\partial \delta u_{i} / \partial x_{j}\right\rangle$ $=\delta_{i x} \delta_{j y} \delta \gamma$. The deviation $\delta u_{i}$ consists of the applied displacement change $\delta_{i x} y \delta \gamma$ and the induced deviation $\delta u_{i}^{\prime}$ localized near the slip. However, the contribution from $\delta u_{i}^{\prime}$ vanishes in Eq. (3.16) from the mechanical equilibrium condition (3.7). Thus,

$$
\frac{\partial}{\partial \gamma} F_{\operatorname{slip}}(\ell, \gamma)=\int d \boldsymbol{r}\left[\sigma_{x y}-\sigma_{x y}^{0}\right]
$$

In Fig. $8 \gamma$ is in the range $|\gamma| \lesssim 0.1$, so $\sigma_{x y}^{0} \cong \gamma$ holds excellently for our elastic energy. Similarly, the counterpart of Eq. (3.17) in the case of uniaxial stretching is written as

$$
\frac{\partial}{\partial \boldsymbol{\epsilon}} F_{\text {slip }}(\ell, \epsilon)=\frac{1}{2} \int d \boldsymbol{r}\left[\sigma_{x x}-\sigma_{y y}-\sigma_{x x}^{0}+\sigma_{y y}^{0}\right]
$$

Relations (3.17) and (3.18) hold for any strain amplitudes as long as a steady slip solution can be obtained from Eq. (3.6), while Eq. (3.12) or Eq. (3.13) is valid only for very small strains $(\lessgtr 0.05$ in our case).
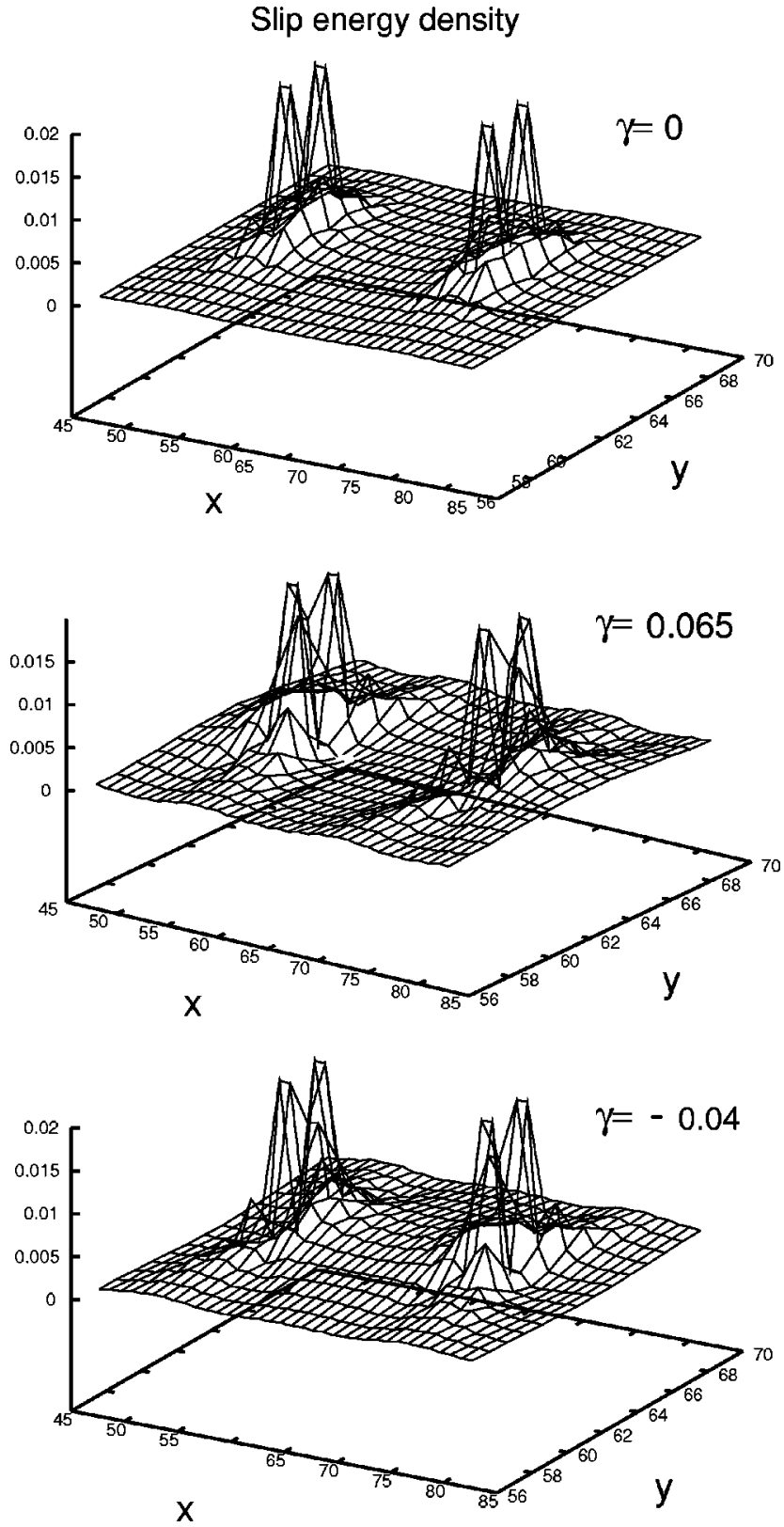

FIG. 7. The slip energy density around a type $C$ slip with length 20 for $\gamma=0$ in (a), 0.065 in (b), and -0.04 in (c). In the middle region between the dislocations at the ends, the elastic energy is decreased in (b) and increased in (c).

\section{Peierls potential energy}

We continue to consider a slip along the $x$ axis under simple shear deformation, but the slip length $\ell$ here can be noninteger. For general $\ell$ we modify Eq. (3.12) as

$$
F_{\mathrm{slip}}=\frac{\ln \ell}{2 \pi(1-\nu)} \mp \gamma \ell+U_{\mathrm{PN}}(\ell)
$$

where $U_{\mathrm{PN}}(\ell)$ represents the Peierls potential energy being zero for integer $\ell$ and nonvanishing for noninteger $\ell$. The force acting on the slip along the glide direction is then given by 


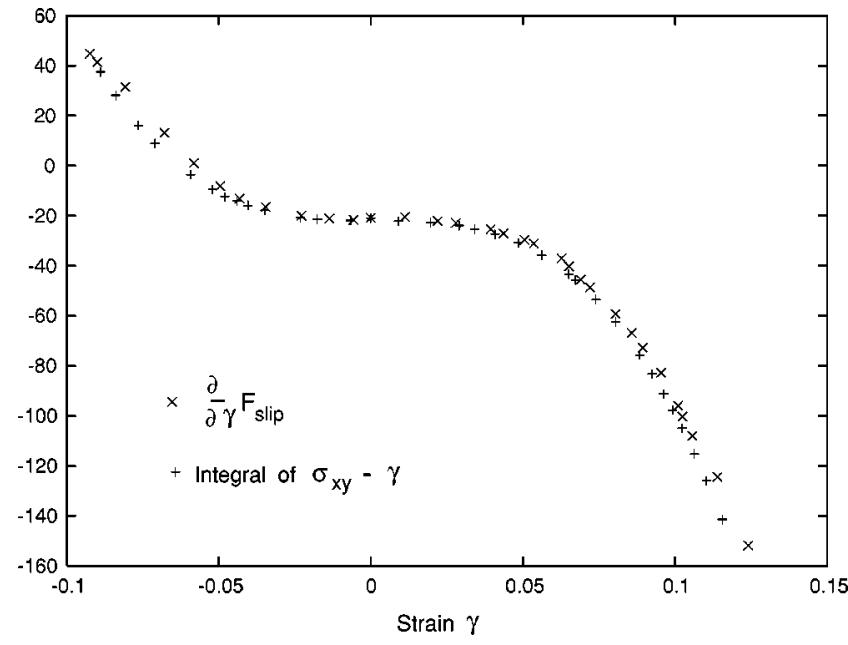

FIG. 8. Coincidence of the derivative $\partial F_{\text {slip }} / \partial \gamma(\times)$ and the space integral of $\sigma_{x y}-\gamma(+)$ confirming the general relation (3.17). These quantities are calculated from the steady slip solutions.

$$
\frac{\partial}{\partial \ell} F_{\text {slip }}=[2 \pi(1-\nu)]^{-1} \frac{1}{\ell} \mp \gamma+\frac{\partial}{\partial \ell} U_{\mathrm{PN}}(\ell) .
$$

This force should vanish for a stationary slip. In the realm of linear elasticity theory, where the crystal structure is smoothed out, the Peierls potential energy is nonexistent and we are led to the following conclusion: A type $\mathrm{CC}$ slip shrinks for any $\ell(>1)$, but a type $C$ slip shrinks for $\ell<\ell_{c}^{\mathrm{L}}$ $=1 /[2 \pi(1-\nu) \gamma]$ and expands for $\ell>\ell_{c}^{\mathrm{L}}$. Here we assume $\gamma>0$. If $\ell$ is fixed, we obtain a critical shear strain $\gamma_{c}^{\mathrm{L}}$ $=1 /[2 \pi(1-\nu) \ell]$. In our nonlinear theory, however, this critical length (or strain) loses its physical relevance.

In our simulations (without the random stress) slips can be stationary suggesting the existence of the Peierls potential energy. To show this, we numerically create two type $C$ slips along the $x$ axis obtained in limit (3.6); one is in the range $0 \leqslant x \leqslant 20$ with length 20 , and the other is in the range -1 $\leqslant x \leqslant 20$ with length 21 . Here the positions of the dislocation core on the left are different by 1 but those on the right coincide. As a result, the corresponding displacements $\boldsymbol{u}_{20}$ and $\boldsymbol{u}_{21}$ are different only near the core region on the left, as can be seen in the inset of Fig. 9. Note that the difference $\boldsymbol{u}^{\prime}=\boldsymbol{u}_{21}-\boldsymbol{u}_{20}$ is the displacement realized when the shorter slip grows into the longer one. Now, we calculate $F$ for the interpolated displacement,

$$
\boldsymbol{u}_{\ell}=(1-\alpha) \boldsymbol{u}_{20}+\alpha \boldsymbol{u}_{21}=\boldsymbol{u}_{20}+\alpha \boldsymbol{u}^{\prime}
$$

As the slip energy at $\ell=20+\alpha(0<\alpha<1), F_{\text {slip }}(\ell)=F$ $-F_{0}$ is determined as in Eq. (3.9). The $F_{\text {slip }}(\ell)$ here depends on the displacement path connecting $\mathbf{u}_{20}$ and $\mathbf{u}_{21}$. In Fig. 9 the resultant energy difference $\Delta F_{\text {slip }}=F_{\text {slip }}(\ell)-F_{\text {slip }}(20)$ is shown. The Peierls potential is determined by $U_{\mathrm{PN}}(\ell)$ $=F_{\text {slip }}(\ell)-[\ln \ell / 2 \pi(1-\nu) \mp \gamma \ell]$ from Eq. (3.19). We recognize that $F_{\text {slip }}(\ell)$ takes a maximum at $\ell=\ell_{\text {max }}$ between 20 and 21 and takes local minima at $\ell=20$ and 21 . It follows that the stable force-balance condition $\partial F_{\text {slip }} / \ell=0$ with

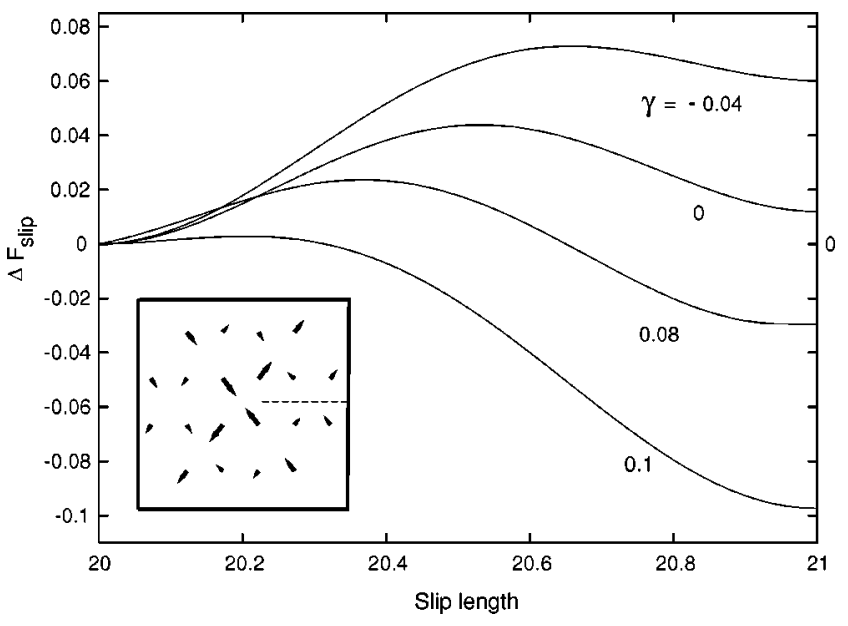

FIG. 9. The slip energy difference $\Delta F_{\text {slip }}(\ell)=F_{\text {slip }}(\ell)$ $-F_{\text {slip }}(20)$ in the range $20 \leqslant \ell \leqslant 21$ obtained for the extrapolated displacement (3.21) for $\gamma=-0.04,0,0.08$, and 0.1 . The position of the maximum is a decreasing function of $\gamma$. The maximum position approaches $\ell=21$ as $\gamma \rightarrow \gamma_{\mathrm{c} 1} \cong-0.09$ and $\ell=20$ as $\gamma \rightarrow \gamma_{\mathrm{c} 2}$ $\cong 0.12$. The inset shows the displacement vector $\boldsymbol{u}_{21}-\boldsymbol{u}_{20}$ needed for slip growth by unit length from $\ell=20$ to 21 .

$\partial^{2} F_{\text {slip }} / \ell^{2}>0$ holds at $\ell=20$ and 21 . The maximum of $U_{\mathrm{PN}}$ is about 0.03 for $\gamma=0$. Figure 9 also indicates that $\ell_{\max }$ $\rightarrow 21 \quad$ as $\quad \gamma \rightarrow \gamma_{\mathrm{c} 1}(\cong-0.09) \quad$ and $\quad \ell_{\max } \rightarrow 20 \quad$ as $\quad \gamma \rightarrow$ $\gamma_{\mathrm{c} 2}(\cong 0.12)$. For integer $\ell$ and noninteger $\ell^{\prime}$, the critical strains, $\gamma_{\mathrm{c} 1}(\ell)$ and $\gamma_{\mathrm{c} 2}(\ell)$, with respect to shrinkage and expansion satisfy the following: (i) $\partial F_{\text {slip }}\left(\ell^{\prime}\right) / \partial \ell^{\prime}>0$ for any $\ell^{\prime}$ smaller than $\ell$ for $\gamma<\gamma_{\mathrm{c} 1}(\ell)$, (ii) $\partial F_{\text {slip }}\left(\ell^{\prime}\right) / \partial \ell^{\prime}$ $<0$ for any $\ell^{\prime}$ larger than $\ell$ for $\gamma>\gamma_{\mathrm{c} 2}(\ell)$, and (iii) $F_{\text {slip }}(\ell)$ is locally minimum at integer length $\ell$ for $\gamma_{\mathrm{c} 1}(\ell)<\gamma$ $<\gamma_{\mathrm{c} 2}(\ell)$. These are consistent with the positions of the instability points in Fig. 6.

\section{PLASTIC FLOW}

In this section we induce deformation at a constant strain rate, $\dot{\gamma}$ or $\dot{\epsilon}$, or cyclic shear deformation for $t \geqslant 0$ in the presence of the random stress tensor $\overleftrightarrow{\sigma}_{\mathrm{R}}$ with $\epsilon_{\mathrm{th}}=0.1$ (except for the curve (b) in Fig. 21 where $\epsilon_{\mathrm{th}}=0.25$ ). At $t=0$, the values of $\boldsymbol{v}$ at the lattice points are Gaussian random numbers with variance 0.01 . The shear stress and the normal stress difference in the following figures are the space averages $\left\langle\sigma_{x y}\right\rangle$ and $\left\langle\sigma_{x x}-\sigma_{y y}\right\rangle$, respectively.

\section{A. Shear deformation}

In Fig. 10 we show the stress-strain curves obtained by integration of Eqs. (2.16) and (2.17) under a constant shear rate $\dot{\gamma}$. At small $\gamma(t)=\dot{\gamma} t$ these curves first follow the curve in the homogeneous case,

$$
\sigma_{x y} \cong A_{3}(\gamma)=\frac{1}{\sqrt{3} \pi} \sin (\sqrt{3} \pi \gamma)
$$

where $A_{3}$ was introduced in Eq. (2.15). For the curve of $\dot{\gamma}$ $=10^{-4}$ and that of $\dot{\gamma}=10^{-3}$ (with the higher peak) we set 


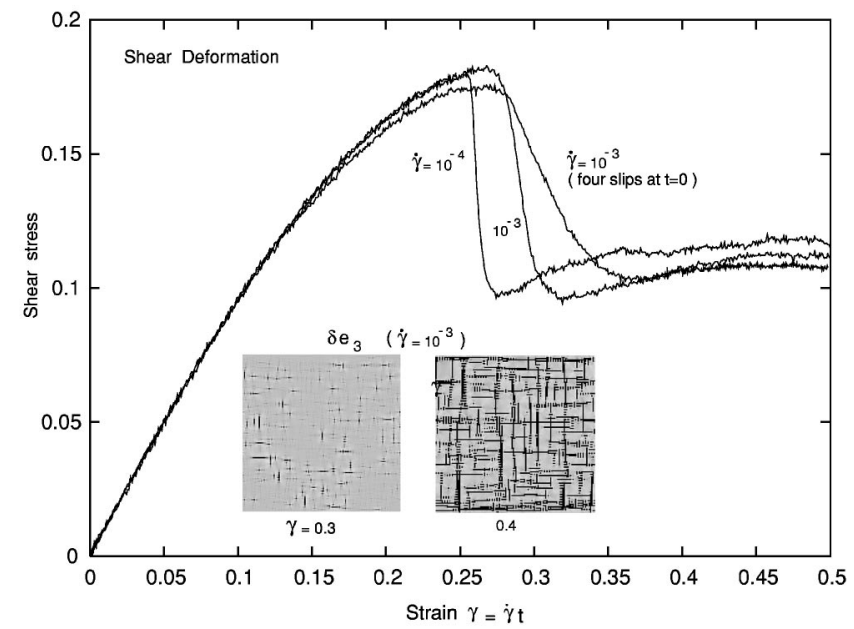

FIG. 10. The stress-strain curves in shear flow. The initial states are defectless for $\dot{\gamma}=10^{-4}$ and $10^{-3}$ (with the higher peak). Four slips are initially prepared for $\dot{\gamma}=10^{-3}$ (with the lower peak). Embedded are snapshots of $\delta e_{3}=e_{3}-\gamma$ at $\gamma=0.3$ at the inception of slip formation and at $\gamma=0.4$ in the plastic flow regime.

$\boldsymbol{u}=\mathbf{0}$ at $t=0$ (supposing a perfect crystal). They approach the elastic instability point $\gamma=\sqrt{3} / 6 \cong 0.289$, where $\partial \sigma_{x y} / \partial \gamma$ tends to vanish and softening with respect to further shear deformation occurs. Then the shear stress drops sharply after the peak with catastrophic formation of slips. See the two snapshots of $\delta e_{3}=e_{3}-\gamma$ in the figure. The orientations of the slips are the most favorable ones with $\varphi=0$ and $\pi / 2$ as determined in Eq. (3.14). For $\dot{\gamma}=10^{-4}$ the slip formation is triggered at a smaller strain $(\cong 0.25)$ and the spacing between the slips is a few times wider than in the case of $\dot{\gamma}$ $=10^{-3}$. In our simulation the slip spacing depends on the shear rate $\dot{\gamma}$ in the plastic flow regime if the other dimensionless parameters are held fixed. For the other curve of $\dot{\gamma}$ $=10^{-3}$ (with the lower peak) we put four slips with length 20 at $t=0$, as will be illustrated in Fig. 13 below in detail. This curve indicates that the overshoot in the stress-strain relation is weakened by the initially preexisting defects. In Fig. 11 we display mechanically unstable points (dots) for $\gamma=0.29,0.30$, and 0.40 at $\dot{\gamma}=10^{-3}$, where at least one of the eigenvalues $\lambda_{1}$ and $\lambda_{2}$ of the matrix $\left\{\Phi_{\alpha \beta}\right\}$ defined below Eq. (2.7) is negative. We recognize that the stability conditions $\left(\lambda_{1}>0\right.$ and $\left.\lambda_{2}>0\right)$ are satisfied at most points in plastic flow. Figure 12 displays a snapshot of the displacement vector $\boldsymbol{u}$ in a $1 / 4$ region of the total system at $\gamma=0.4$ with $\dot{\gamma}=10^{-4}$. We can see a number of slips and bands (aggregates of slips here), where $\varphi=0$ for type $C$ and $\varphi=\pi / 2$ for type CC. The large horizontal shear band in the lower part is particularly conspicuous, where the band thickness and the discontinuity of $u_{x}$ across it are both increased up to about three to four. We recognize that elementary slips (dipoles of edge dislocations) tend to be created around preexisting ones, yielding thicker shear bands. Similar thick shear bands have been observed in previous simulations $[17,18]$.

In Fig. 13 we follow time-development of $\delta e_{3}$ and $\delta f_{\text {el }}$ $=f_{\mathrm{el}}-\left\langle f_{\mathrm{el}}\right\rangle$ at $\dot{\gamma}=10^{-3}$ in the presence of four slips at

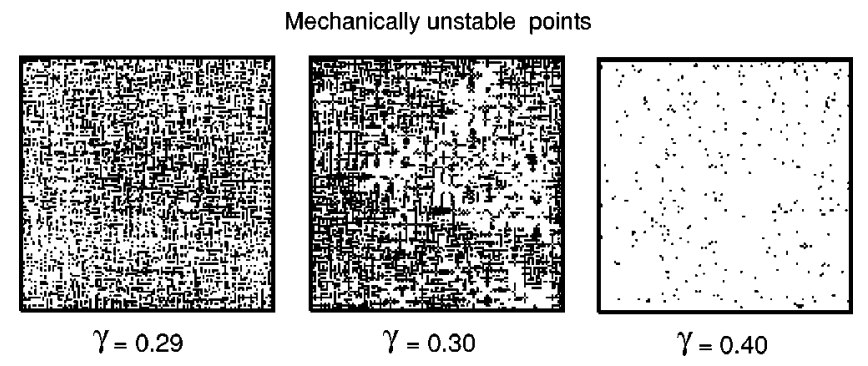

FIG. 11. Mechanically stable regions (white) and unstable points (black) after application of shear flow with $\dot{\gamma}=10^{-3}$. The initial state is crystalline without defects. At the stable points the eigenvalues of the matrix $\left\{\Phi_{\alpha \beta}\right\}$ defined below Eq. (2.7) are both positive, while at the unstable points at least one of them is negative. At $\gamma=0.29$ the system is close to the peak position, at $\gamma$ $=0.30$ slips are appearing, and at $\gamma=0.4$ the unstable points are fluctuating in time near the dislocation cores.

$t=0$, where the favorable slips (in black in the upper part at $\gamma=0$ ) grow and the unfavorable ones (in white) shrink as $\gamma$ increases. In the lower snapshots the elastic energy density deviation $\delta f_{\mathrm{el}}=f_{\mathrm{el}}-\left\langle f_{\mathrm{el}}\right\rangle$ is shown, where the black dots represent the dislocation cores. At $\gamma=0.176$ we can see that the energy density between the two dislocations at the slip ends is decreased for the favorable slips and is increased for the unfavorable slips, in accord with Eq. (3.13) and Fig. 7. At the plastic flow regime $\gamma=0.387$ the initial favorable slips grow into thick layers where the dislocation density is very high.

Next we apply a cyclic shear deformation, where $\dot{\gamma}(t)$ $=10^{-3}$ in the time regions $n t_{\mathrm{p}}<t<(n+1 / 2) t_{\mathrm{p}}$ and

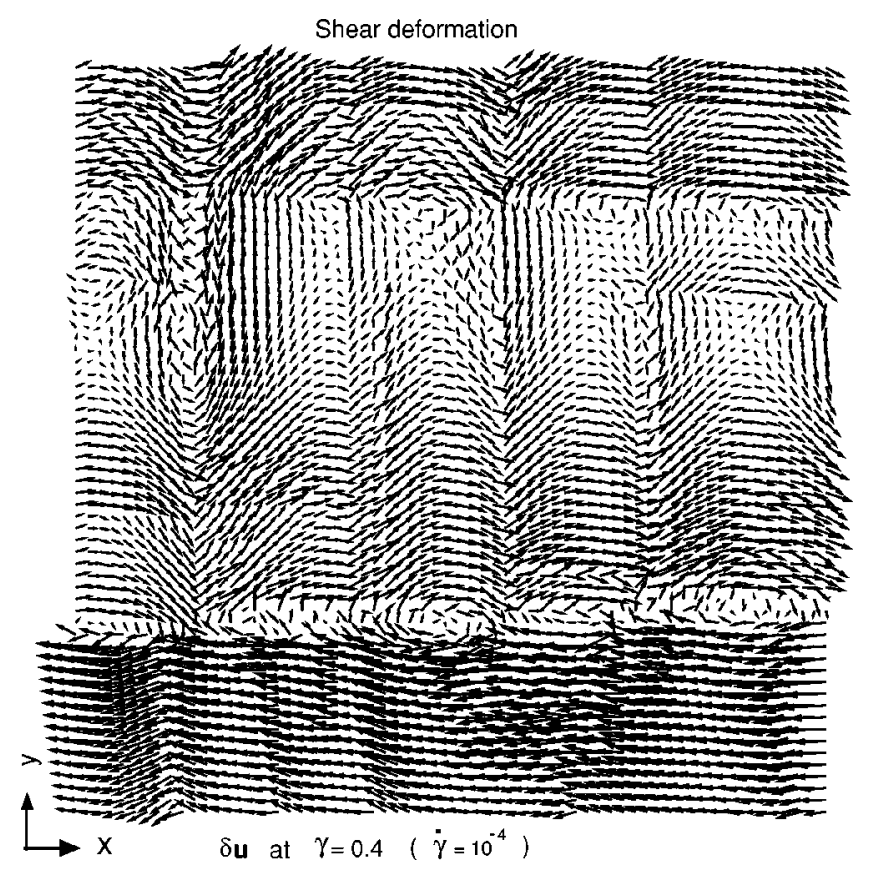

FIG. 12. The displacement deviation $\delta \boldsymbol{u}=\boldsymbol{u}-(\gamma y, 0)$ in the plastic flow regime under shear strain at $\gamma=0.4$ with $\dot{\gamma}=10^{-4}$. A $1 / 4$ region $(64 \times 64)$ of the total system is shown. The arrows are from the original position at $t=0$ in a perfect crystal to the displaced position in plastic flow. 
Shear deformation $\gamma=\dot{\gamma} \mathrm{t}$

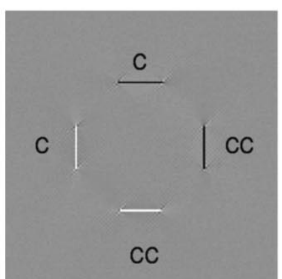

$\gamma=0$

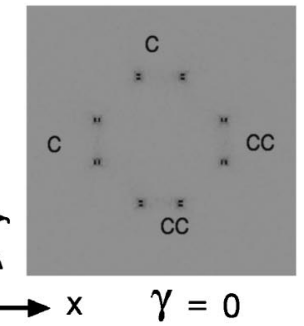

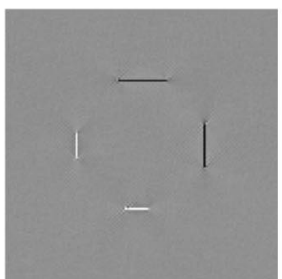

0.176

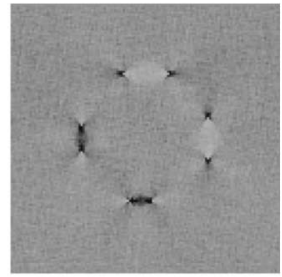

0.176

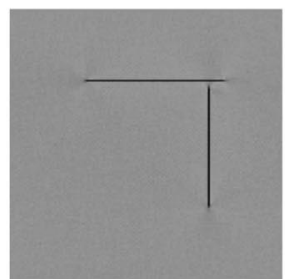

0.25

$\delta e_{3}$

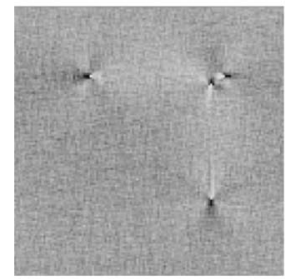

0.25

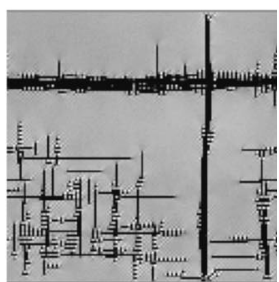

0.387

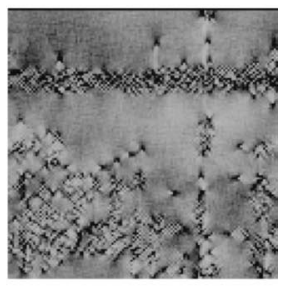

0.387
FIG. 13. Time evolution of $\delta e_{3}$ and $\delta f_{\text {el }}$ in shear flow when four slips are placed in the initial state. $\dot{\gamma}(t)=-10^{-3}$ in the time regions $(n+1 / 2) t_{\mathrm{p}}<t<(n$ $+1) t_{\mathrm{p}}$. We choose $t_{\mathrm{p}}=1000$, so the maximum of $\gamma(t)$ is 0.5 and the minimum is 0 . For the first two cycles $(n=0$ and 1$)$, Fig. 15 shows the stress-strain curve, while Fig. 16 shows the average elastic energy density $\left\langle f_{\mathrm{el}}\right\rangle$ with a snapshot of $f_{\mathrm{el}}$ at the point $A$ where the average stress vanishes. As salient features, we notice (i) residual strain at vanishing stress, (ii) the shear stress becomes negative at the end of the first cycle, (iii) no overshoot in the stress and the elastic energy from the second cycle, and (iv) that the stress and the elastic energy take roughly constant values characteristic of well-developed plastic flow in the region $0.25 \leqslant \gamma(t)<0.5$ for increasing $\gamma(t)$ and in the region $0<\gamma(t) \lesssim 0.25$ for decreasing $\gamma(t)$. Thus we can see significant hysteresis behavior. In MD simulations of low $T$ glasses, similar stress-strain curves under stepwise strain rates have been obtained (but without overshoot behavior because of disordered initial states) $[16,41]$.

We also notice that at the points $A, B$, and $C$, where the average stress vanishes as in Fig. 14, the curves of $\left\langle f_{\text {el }}\right\rangle$ in Fig. 15 are locally minimum. This suggests that the strain $\gamma(t)$ consists of an elastic strain $\gamma_{\mathrm{el}}$ and a slip strain

$$
\gamma_{\mathrm{s}}=\gamma-\gamma_{\mathrm{el}}
$$

Roughly speaking, the elastic strain outside the slip lines should give rise to the average stress, while the slip strain is caused by the jumps of $u_{x}$ across the slips. To be more quantitative, we define $\gamma_{\mathrm{el}}$ as

$$
A_{3}\left(\gamma_{\mathrm{el}}\right)=\left\langle\sigma_{x y}\right\rangle
$$

The elastic energy density stored is then the sum of the elastic energy density and the defect energy density. We define the average defect energy density by

$$
f_{\mathrm{D}}=\left\langle f_{\mathrm{el}}\right\rangle-\Phi\left(\gamma_{\mathrm{el}}, 0\right)
$$

where $\Phi$ is defined by Eq. (2.5). For small $\gamma_{\mathrm{el}}$ we have $\gamma_{\mathrm{el}}$ $\cong\left\langle\sigma_{x y}\right\rangle$ and $\Phi\left(\gamma_{\mathrm{el}}, 0\right) \cong \gamma_{\mathrm{el}}^{2} / 2$. Hence, in the vicinity of the points where $\left\langle\sigma_{x y}\right\rangle=0,\left\langle f_{\text {el }}\right\rangle(t)$ should take a minimum, provided that $f_{\mathrm{D}}(t)$ changes slowly there. To confirm these arguments, we plot $f_{\mathrm{D}}(t)$ in Fig. 16. For $\gamma(t) \lesssim 0.3$ in the first cycle, however, it represents the elastic energy due to the inhomogeneous fluctuations of the local strains (mainly due to $\delta e_{3}$ ) with the peak height at 0.012 (not shown in the figure). After this initial period, $f_{\mathrm{D}}(t)$ is in a range of $0.004-0.005$ reasonably representing the elastic energy due to the defects produced in plastic flow. The energy variance $\sqrt{\left\langle\left(\delta f_{\text {el }}\right)^{2}\right\rangle}$ is also about 0.005 in plastic flow obviously due to the discrete nature of dislocation cores. For $\dot{\gamma}=10^{-4}$, on the other hand, $f_{\mathrm{D}}(t)$ is almost constant around 0.002 in plastic flow.

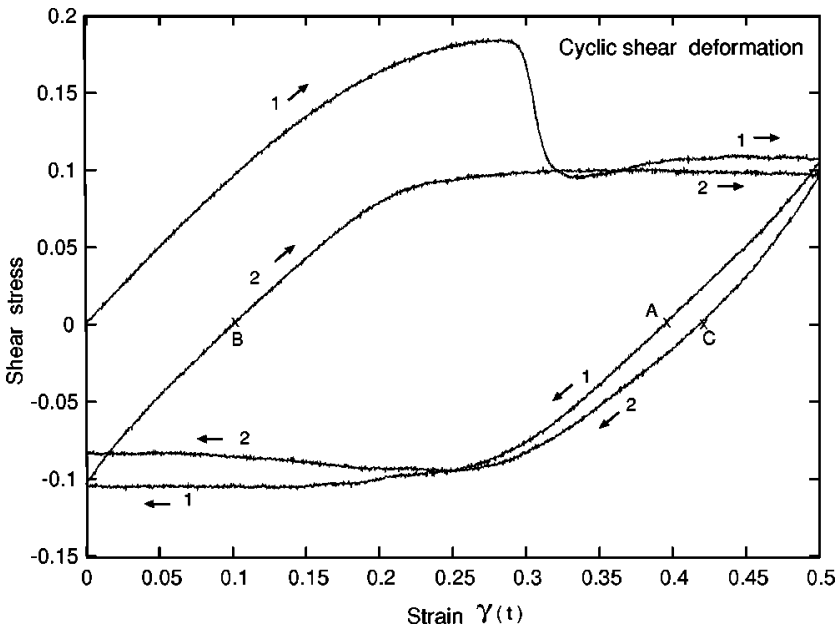

FIG. 14. The stress-strain curve for cyclic shear deformation in the first two cycles at $\dot{\gamma}= \pm 10^{-3}$ with period $10^{3}$. Once $\gamma \geq 0.3$ in the first cycle, high-density dislocations are created. The shear stress vanishes at the three points $A, B$, and $C(\times)$. 


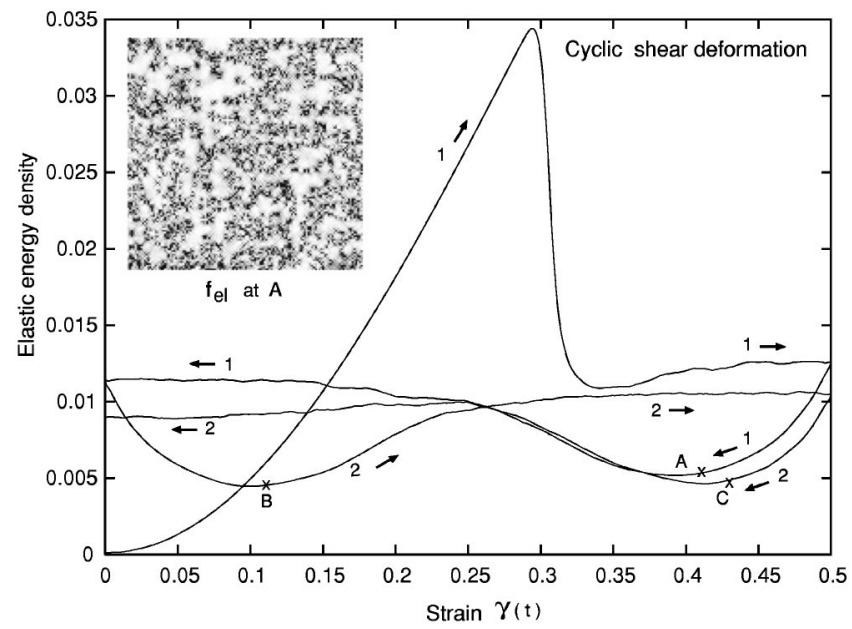

FIG. 15. The average elastic energy density $\left\langle f_{\text {el }}\right\rangle$ vs the strain $\gamma(t)$ for cyclic shear deformation in the first two cycles. At the points $A, B$, and $C$ the shear stress vanishes. In the inset the snapshot of $f_{\mathrm{el}}$ at the point $A$ is shown, where $\left\langle\sigma_{x y}\right\rangle=\gamma_{\mathrm{el}}=0$ and the black points represent dislocation cores.

\section{B. Uniaxial stretching}

In Fig. 17 we show the normal stress vs strain at constant strain rate $\dot{\epsilon}$ for $t \geqslant 0$. The characteristic features are very similar to those in Fig. 10. At small $\epsilon(t)=\dot{\epsilon} t$ these curves first follow the curve in the homogeneous case,

$$
\sigma_{x x}-\sigma_{y y} \cong 2 A_{2}(\epsilon)=\frac{2}{3 \pi}[\sin (\pi \epsilon)+\sin (2 \pi \epsilon)],
$$

where $A_{2}$ was introduced in Eq. (2.15). For the curve of $\dot{\epsilon}$ $=10^{-4}$ and that of $\dot{\epsilon}=10^{-3}$ (with the higher peak) we set $\boldsymbol{u}=\mathbf{0}$ at $t=0$. They approach the elastic instability point $\boldsymbol{\epsilon}$ $=\pi^{-1} \cos ^{-1}[(\sqrt{33}-1) / 8] \cong 0.298$. After the catastrophic formation of slips, the slip orientation angles are $\pm \pi / 4$ with

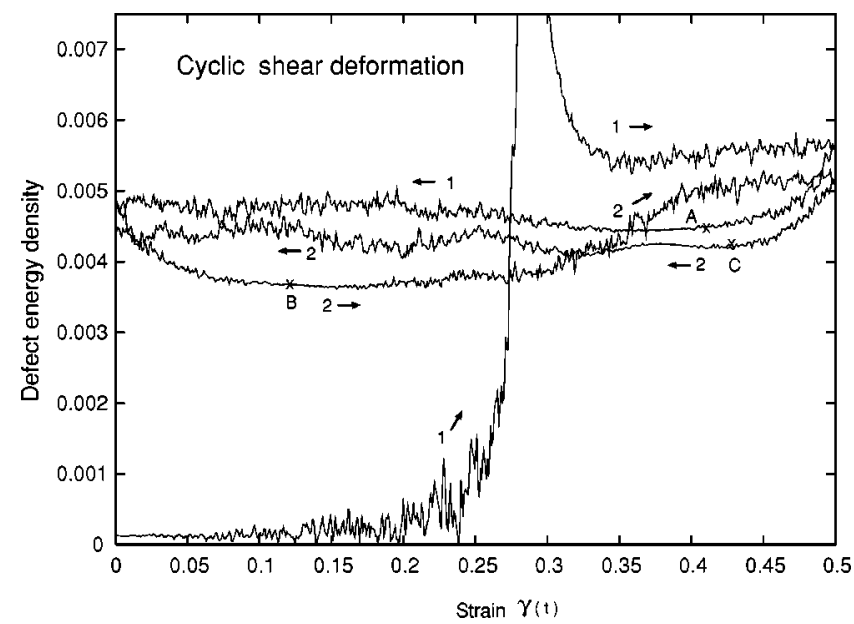

FIG. 16. The energy density $f_{\mathrm{D}}$ defined by Eq. (4.2) vs the strain $\gamma(t)$ for cyclic shear deformation in the first two cycles. It is the defect energy density in plastic flow, but in the preplastic regime it arises from the heterogeneities in the strains and is enhanced at the onset of plastic flow.

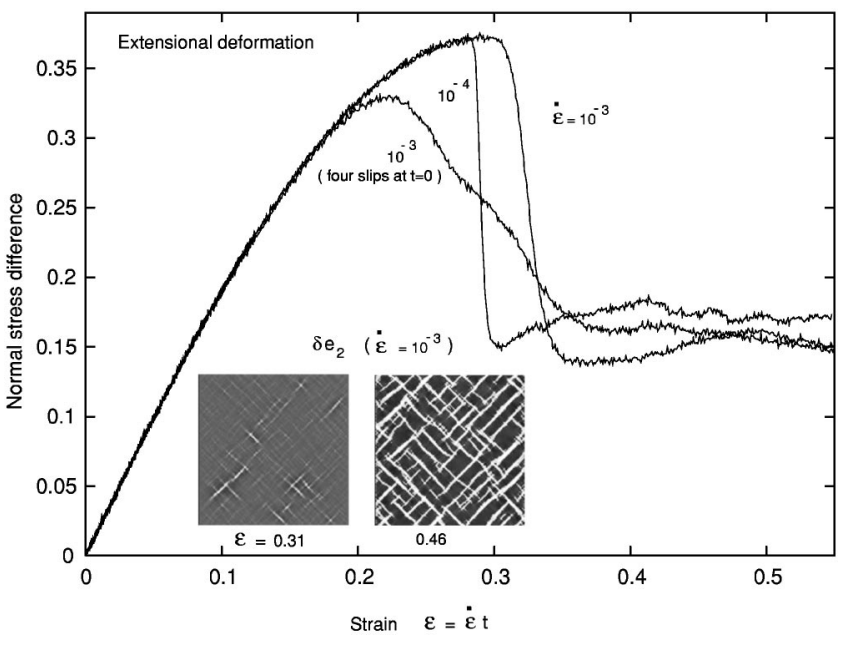

FIG. 17. The stress-strain curves under uniaxial stretching. The initial states are defectless for $\epsilon=10^{-4}$ and $10^{-3}$ (with the higher peak). Four slips are initially prepared for $\epsilon=10^{-3}$ (with the lower peak). Embedded are snapshots of $\delta e_{2}=e_{2}-\epsilon$ at $\epsilon=0.31$ at the inception of slip formation and at $\epsilon=0.46$ in the plastic flow regime.

respect to the stretched direction in agreement with the experiments $[8-11,29,36,37]$ and the simulations $[17,18]$. The discontinuity across the slip lines appears in the tetragonal strain $e_{2}$, which can be seen in the two snapshots of $\delta e_{2}$ $=e_{2}-\epsilon$. In Fig. 18 the displacement vector $\boldsymbol{u}$ in a 1/4 region of the total system at $\epsilon=0.46$ with $\dot{\epsilon}=10^{-3}$ is displayed, where the orientations of the slips are the most favorable

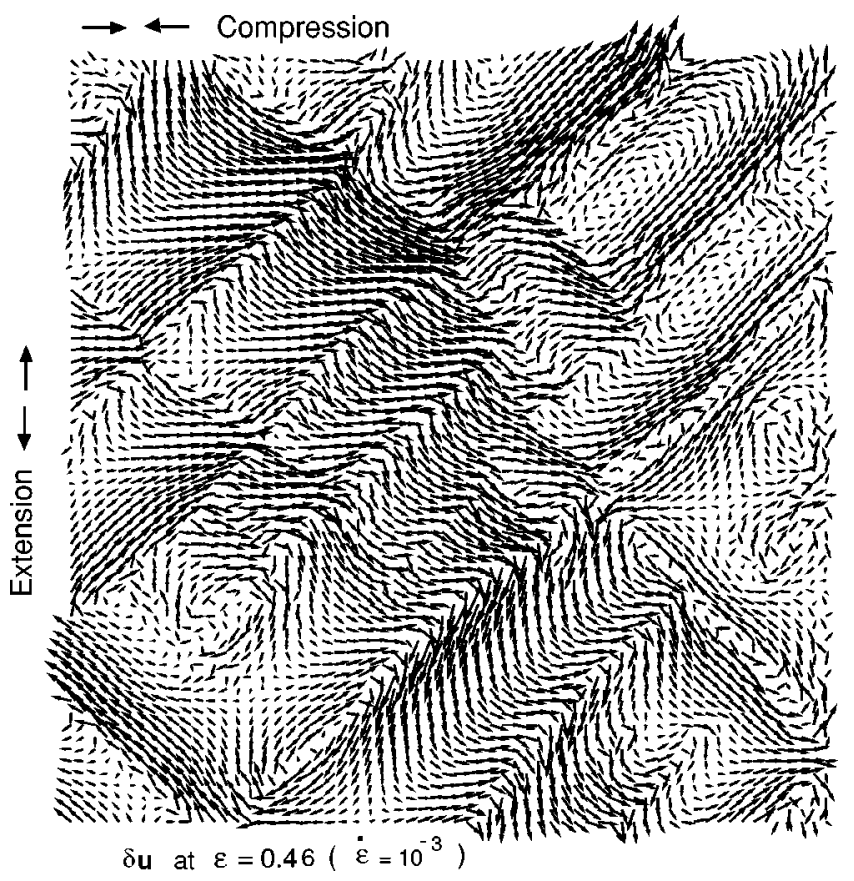

FIG. 18. The displacement deviation $\delta \boldsymbol{u}=\boldsymbol{u}-(\boldsymbol{\epsilon} x / 2,-\boldsymbol{\epsilon} / 2)$ in the plastic flow regime under uniaxial stretching at $\epsilon=0.46$ with $\epsilon=10^{-3}$. A $1 / 4$ region $(64 \times 64)$ of the total system is shown. The arrows are from the original position at $t=0$ in a perfect crystal to the displaced position in plastic flow. 


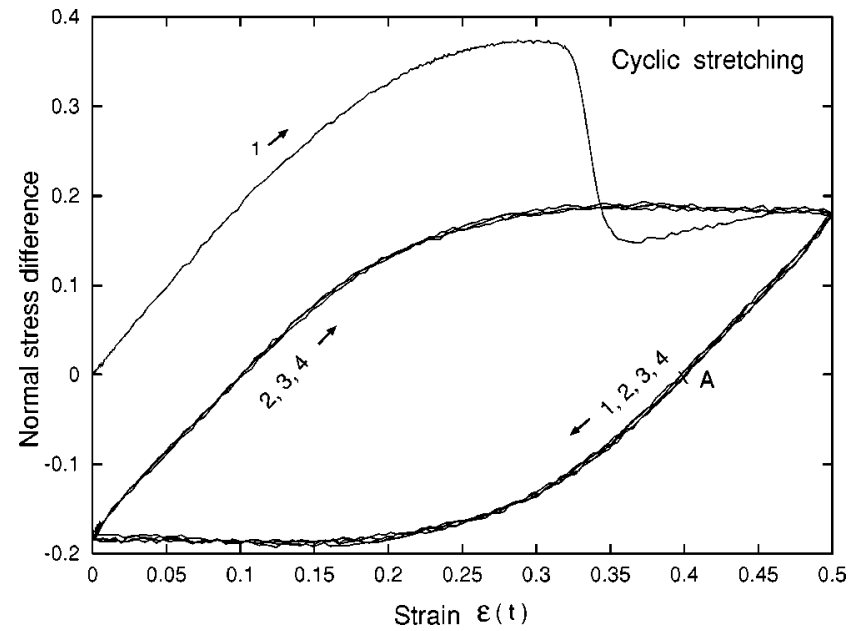

FIG. 19. The stress-strain curve for cyclic uniaxial stretching in the first four cycles at $\dot{\epsilon}= \pm 10^{-3}$ with period $10^{3}$. Once $\epsilon \gtrsim 0.3$ in the first cycle, high-density dislocations are created. In this case the stress-strain relation becomes nearly periodic from the second cycle.

ones with $\varphi= \pm \pi / 4$ as determined below Eq. (3.14). In the plastic flow regime, the shear bands grow into thick layers containing high-density dislocations.

Next we apply cyclic uniaxial stretching, where $\dot{\epsilon}(t)$ $=10^{-3}$ in the time regions $n t_{\mathrm{p}}<t<(n+1 / 2) t_{\mathrm{p}}$ and $\dot{\epsilon}(t)$ $=-10^{-3}$ in the time regions $(n+1 / 2) t_{\mathrm{p}}<t<(n+1) t_{\mathrm{p}}$. We choose $t_{\mathrm{p}}=1000$, so the maximum of $\epsilon(t)$ is 0.5 and the minimum is 0 . Figure 19 shows the stress-strain curve for the first four cycles, where it is nearly periodic from the second cycle. As in the shear deformation case we introduce the average elastic strain $\epsilon_{\mathrm{el}}$ by

$$
2 A_{2}\left(\epsilon_{\mathrm{el}}\right)=\left\langle\sigma_{x x}-\sigma_{y y}\right\rangle
$$

and the average defect energy density $f_{\mathrm{D}}$ by

$$
f_{\mathrm{D}}=\left\langle f_{\mathrm{el}}\right\rangle-\Phi\left(0, \epsilon_{\mathrm{el}}\right)
$$

where $\Phi$ is defined by Eq. (2.5). The average slip strain is given by $\epsilon_{\mathrm{s}}=\epsilon-\epsilon_{\mathrm{el}}$. For small $\epsilon_{\mathrm{el}}$ we have $2 \epsilon_{\mathrm{el}}$ $\cong\left\langle\sigma_{x x}-\sigma_{y y}\right\rangle$ and $\Phi\left(0, \epsilon_{\mathrm{el}}\right) \cong \epsilon_{\mathrm{el}}^{2} / 2$. Figure 20 shows the average elastic energy density $\left\langle f_{\text {el }}\right\rangle$ and the defect energy density $f_{\mathrm{D}}$. The latter is in a range $0.008-0.009$ in plastic flow. The inset of Fig. 20 displays a snapshot of $f_{\mathrm{el}}$ at the point $A$ in the first cycle, where the average normal stress difference vanishes. Comparing it with the snapshot of $f_{\mathrm{el}}$ in Fig. 15 under shear deformation, we notice a considerable difference in the spatial anisotropy of the dislocation distribution between the two cases.

\section{Strain-induced disordered states}

In the plastic flow regime dislocations are proliferated and a structurally disordered state is realized. This effect may be called strain-induced disordering. We mention a simulation by Ikeda et al. [57], who applied a tensile strain to a 3D model to induce a change from a perfect crystal to an amor-

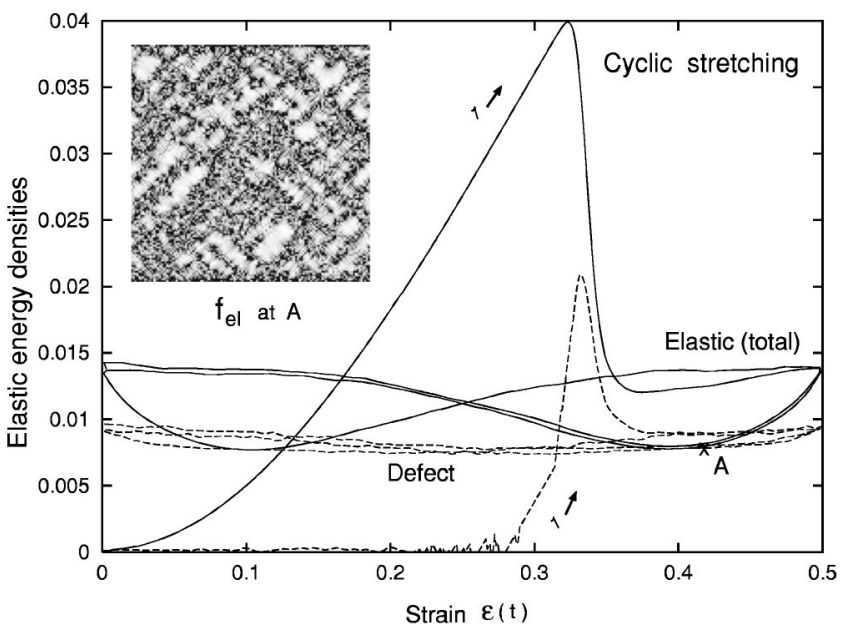

FIG. 20. The average total elastic energy density $\left\langle f_{\text {el }}\right\rangle$ (solid line) and the defect part $f_{\mathrm{D}}$ (broken line) vs the strain $\epsilon(t)$ for cyclic uniaxial stretching in the first two cycles. In the inset the snapshot of $f_{\text {el }}$ at the point $A$ in the first cycle in Fig. 19 is shown, where $\left\langle\sigma_{x x}-\sigma_{y y}\right\rangle=\epsilon_{\mathrm{el}}=0$ and the black points represent dislocation cores.

phous solid. In Fig. 4 in our previous work [39] we switched off a shear flow (a) before the peak time of the stress, (b) just after the peak time, and (c) in well-developed plastic flow. Affine deformation in (a) was maintained, while no appreciable time evolution was detected after transients in (b) and (c). This means that the structurally disordered states are metastable obviously due to the Peierls potential.

Here a shear flow with $\dot{\gamma}=10^{-3}$ is applied at $t=0$ and is stopped at time $t_{\mathrm{A}} \cong 600$ (at the point $A$ in Fig. 14), and then the system is relaxed for a time period of $t_{\mathrm{w}}=10^{5}$. In Fig. 21, slow relaxation of the average energy density $\left\langle f_{\text {el }}\right\rangle(t)$ $=F_{\text {el }} / N^{2}$ is shown in the time range $t_{\mathrm{A}}<t<t_{\mathrm{A}}+t_{\mathrm{W}}$, where we set $u_{x}=\gamma_{\mathrm{A}} L_{0}$ and $u_{y}=0$ at the top $y=L_{0}$ with $\gamma_{\mathrm{A}}$ $\cong 0.40$ together with $\boldsymbol{u}=\mathbf{0}$ at the bottom. The dislocation distributions here closely resemble that in the inset of Fig. 15 (but are not identical because they are obtained from different runs). In the upper curve (a) the noise strength $\epsilon_{\text {th }}$ in Eq. (2.25) is 0.1 as in the previous simulations in this section, where each maximum in the initial stage corresponds to an energy increase accompanied with a configuration change around a dislocation core. In (a) the typical energy increase (in $F_{\text {el }}$ ) is of order $10^{-5} N^{2} \sim 0.1$ for each event. However, there is no appreciable relaxation for $t \gtrsim 3 \times 10^{3}$. In (b) we increase the noise strength $\epsilon_{\text {th }}$ to 0.25 to obtain a larger energy decrease in $\left\langle f_{\mathrm{el}}\right\rangle(t)$ with a larger thermal noise superimposed. In these cases only a small number of configuration changes $(\sim 10)$ occur around dislocation cores even for $t_{\mathrm{w}}$ $=10^{5}$, so the effect of the structural relaxation is negligible on the macroscopic level (no aging effect). In fact, the stressstrain curves after switching-on of the shear flow at $t=t_{\mathrm{A}}$ $+t_{\mathrm{w}}$ with $\dot{\gamma}=10^{-3}$ are almost independent of $t_{\mathrm{w}}$ if plotted as a function of $\Delta \gamma(t)=\dot{\gamma}\left(t-t_{\mathrm{A}}-t_{\mathrm{w}}\right)$. Interestingly, they exhibit a rounded peak at $\Delta \gamma=0.15$ as can be seen in the inset. We also comment on the average shear stress $\left\langle\sigma_{x y}\right\rangle(t)$. In the waiting time region $\left(t_{\mathrm{A}}<t<t_{\mathrm{A}}+t_{\mathrm{w}}\right)$, it fluctuates in time 


\section{Energy relaxation at $\mathrm{A}$}

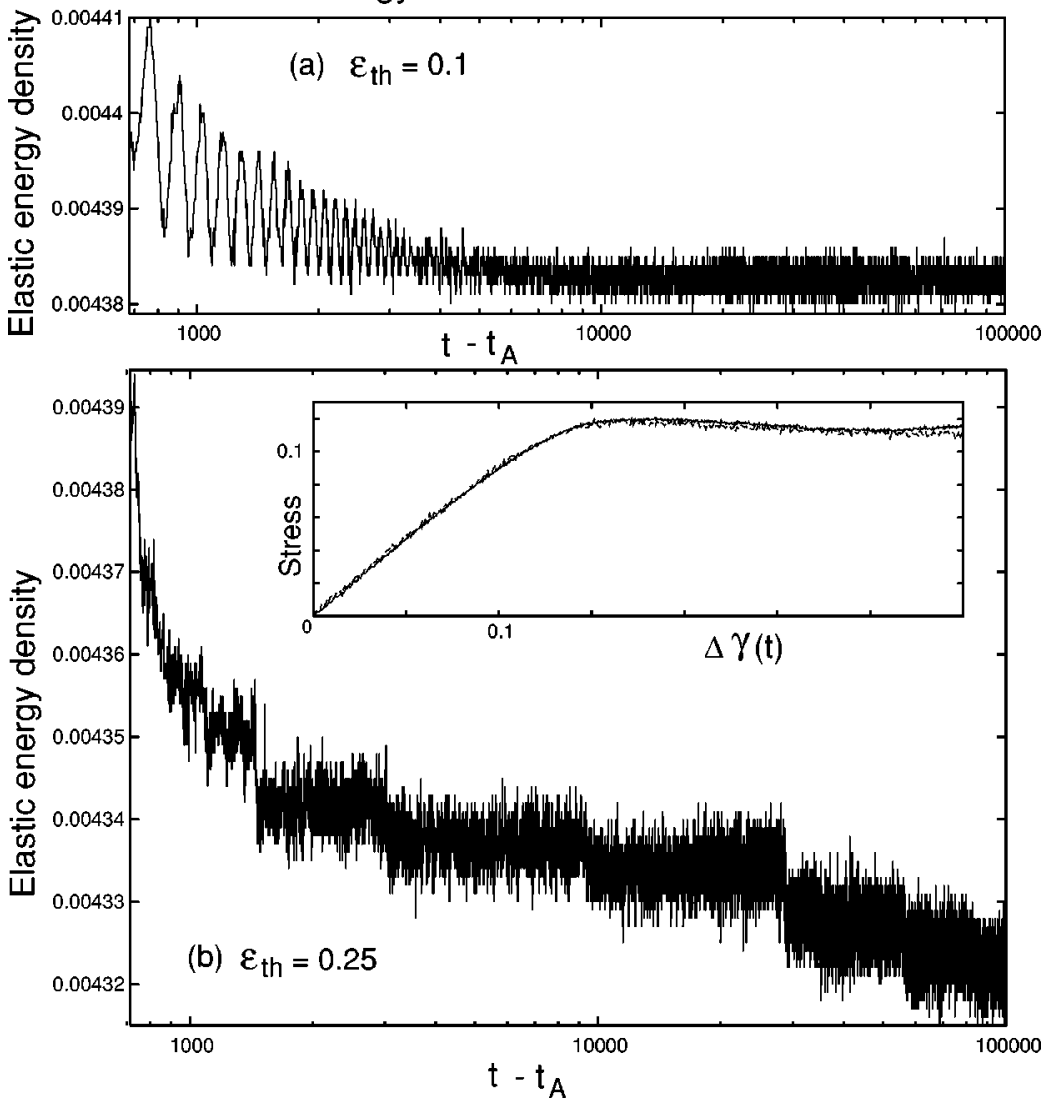

FIG. 21. Relaxation of the average energy density $\left\langle f_{\mathrm{el}}\right\rangle(t)$ for $\epsilon_{\mathrm{th}}=0.1$ (a) (upper curve) and $\epsilon_{\mathrm{th}}=0.25$ (b) (lower curve). The shear flow is stopped at time $t_{\mathrm{A}} \cong 600$ (at the point $A$ in Fig. 14) and the system is relaxed for a time period of $t_{\mathrm{w}}=10^{5}$. The energy decreases are extremely small as compared to the initial values, so there is almost no appreciable aging behavior here. The inset displays the stress-strain curves, $\left\langle\sigma_{x y}\right\rangle$ vs $\Delta \gamma(t)$, after the shear is switched on again for $t$ $\geqslant t_{\mathrm{A}}+t_{\mathrm{w}}$, where the solid line corresponds to (a) and the noisy broken line to (b). The $\Delta \gamma(t)$ $=\dot{\gamma}\left(t-t_{\mathrm{A}}-t_{\mathrm{w}}\right)$ is the excess strain with $\dot{\gamma}$ $=10^{-3}$. Almost identical stress-strain curves are obtained for any waiting time $t_{\mathrm{w}}$ shorter than $10^{5}$. around 0 , and its noise amplitude is only of order 0.002 for (a) and 0.004 for (b), so the stress is nearly fixed.

Note that the crystal angle $\theta$ with respect to the $x$ axis has been assumed to be 0 [see Eq. (2.12)]. We need to show that the strain-induced disordered states remain metastable even for different angles of the crystal axes with respect to the flow direction. To this end, as in Fig. 21(a), we have calculated the elastic energy density for $\theta=\pi / 12$ with the other parameters unchanged for $t<10^{5}$. In such a run, it has decreased from 0.006605 to 0.006575 for $t_{\mathrm{A}}<t \lesssim 3000$ and saturated for $t \gtrsim 3000$, similarly to the behavior in Fig. 21(a), again leading to no appreciable aging.

\section{SUMMARY AND CONCLUDING REMARKS}

In summary, we have presented a nonlinear elasticity theory taking into account the periodicity of the elastic energy density with respect to the shear and tetragonal strains, $e_{3}$ and $e_{2}$. It has the symmetry of the 2D triangular lattice but is surprisingly isotropic in the $e_{3}-e_{2}$ plane. We summarize our main results together with some comments.

(i) We have numerically examined the slip structure as a function of its length $\ell$, its angle $\varphi$, and applied strains $\gamma$ and $\epsilon$. In external strain field, slips should appear in the orientations minimizing the slip energy $F_{\text {slip }}$ and they should grow into shear bands observed in previous experiments under external load in various materials. The snapshots of the displacement vector in the plastic flow regime, Fig. 12 for shear deformation and Fig. 18 for uniaxial stretching, most unambiguously illustrate the physical processes taking place.
We remark that the previous experiments have mostly been performed under uniaxial (biaxial or triaxial) deformation, so future experiments with shear deformation $\left(\left\langle u_{x}\right\rangle=\gamma y\right.$ and $\left\langle u_{y}\right\rangle=0$ ) should be informative. On the other hand, in crystalline solids with strong crystal anisotropy, the slip planes are parallel to particular crystal planes [58]. In our simulations we may obtain slips as steady solutions of our dynamic model due to the presence of the Peierls potential energy. However, they cannot be stationary for $\gamma<\gamma_{\mathrm{c} 1} \sim-0.1$ or $\gamma$ $>\gamma_{\mathrm{c} 2} \sim 0.1$ in shear strain $\gamma$, where the potential minima disappear and the slips shrink or grow.

(ii) We have examined plastic flow by applying a constant strain rate at $t=0$. If there is no initial disorder, the stressstrain curve exhibits a pronounced overshoot with a peak stress of order $10 \%$ of the shear modulus. However, in the presence of initial disorder, the overshoot is weakened or even erased, as revealed by simulations with initial four slips in Fig. 10, under cyclic shear in Figs. 14 and 20, and after staying at the zero-stress point $A$ in the inset of Fig. 21. In accordance with these findings, previous simulations performed with various initial states have demonstrated sensitive dependence of the overshoot behavior on the quenching conditions $[18,59]$. In addition, a number of previous simulations have reported either existence or nonexistence of the stress peak. In some real glassy systems including polymers, overshoot behavior has been widely observed [13,26,27,29]. In an amorphous metal, the stress increased monotonically to a steady-state value for slow strain rate $\left(\dot{\epsilon}<10^{-3} \mathrm{~s}^{-1}\right)$, while a maximum appeared at $\epsilon \sim 0.06$ for large strain rate $\left(\dot{\epsilon}>5 \times 10^{-3} \mathrm{~s}^{-1}\right)[27]$. 
(iii) As illustrated in Figs. 16 and 20 we have divided the total elastic energy into the affine part and the defect part following definitions (4.4) and (4.7). In our model, once defects are created, the defect elastic energy is rather weakly dependent on the deformation history. On the basis of this division, we may easily understand the characteristic features of the stress-strain curves in the cyclic straining mentioned in the preceding section. In future microscopic simulations on glassy materials, this kind of energy division should be informative at relatively small strains, where we are interested in the defect contribution to measurable quantities such as the specific heats. Here we mention microscopic calculations of the average potential energy per particle $\langle e\rangle$ in supercooled states under shear [59-61], where $\langle e\rangle$ was increased considerably by shear flow above the initial value in quiescent states. It is remarkable that, while $\langle e\rangle$ in quiescent states sensitively depends on the quenching history (aging effect) $[59,62]$, it becomes uniquely determined in shear flow for shear rates larger than the inverse structural relaxation time $\tau_{\alpha}^{-1}$. This is because sheared systems are effectively driven away from the glass transition [24]. This effect would be consistent with our result that $f_{\mathrm{D}}(t)$ is kept nearly constant in plastic flow as in Figs. 16 and 20.

(iv) In our simulations slips emerge as long straight lines, as shown in the snapshots of $e_{3}$ or $e_{2}$. In our model the crystal order is not broken over long distances, but if disorder is fully introduced, the glide motions of slips in particular directions should be much limited. In MD simulations of two component glasses, for example, such degree of disorder should be sensitive to the size ratio of the two species. In fact, it was rather close to 1 in the simulation by Deng et al. $[13,16]$, where nanocrystalline order was realized [63] because of rather weak frustration and long slips with atomic thickness emerged along the crystal axes. It is therefore informative to perform MD simulations with various size ratios and examine how the shapes of local configuration changes depend on the size ratio.

(v) As a special ingredient of our theory, our elastic energy is almost isotropic if the distance from the center of a unit cell in the $e_{3}-e_{2}$ plane is shorter than 0.5 (see Figs. 1-3). The snapshot at $\gamma=0.4$ in Fig. 11 demonstrates that most spatial points are in the mechanically stable regions in plastic flow and hence are in the isotropic elasticity regions. Therefore, our results such as the orientations of well-developed shear bands should be applicable to those in amorphous materials (which are isotropic on large scales).

(vi) The dimensionless parameter $\lambda$ in Eq. (2.23) is related to Poisson's ratio $\nu$ as in Eq. (3.2) and the dislocation energy depends on $\nu$. Our choice $K_{0} / \mu_{0}=\lambda=1$ or $\nu=0$ is rather unusual, since $K_{0}$ is usually considerably larger than $\mu_{0}$ in high density systems. The appropriateness of the other choices $\eta_{0}^{*}=1$ in Eq. (2.26) and $\epsilon_{\text {th }}=0.1$ in Sec. IV should also be examined in future.

(vii) As another aspect, we mention the effect of elastic interaction among dislocations. In our case slips are more easily created around preexisting ones, as already reported in Ref. [18]. In the insets of Figs. 15 and 20 we can see a tendency of aggregation of dislocation cores. In real 3D crystals a tangle of dislocations often appears as a characteristic feature of fatigued states [64] and has also been realized in 3D large-scale simulations $[3,4]$. In the literature the interdislocation elastic interaction is believed to yield mesoscopic patterns in the dislocation distribution $[3,4,64]$.

(viii) As already stressed in the Introduction, in order to describe glass dynamics in a more satisfactory level, we should try to include a variable representing the local freevolume [39] and the configurational frustration effect induced by the size disparity between the two species. This generalization should be essential with increasing $T$ towards the glass transition. For example, we may predict a gradual diffusional increase of the free volume around dislocation cores [39], which induces breakage of the Peierls potential and configuration changes presumably resulting in significant aging effects. [The critical strains $\gamma_{\mathrm{c} 1}$ and $\gamma_{\mathrm{c} 2}$ discussed below Eq. (3.21) and indicated in Fig. 6 by arrows are sensitively decreased in magnitude by a small amount of the local free volume near the dislocation cores.] In the present study, as shown in Fig. 21, we have found no appreciable aging effect.

(ix) The simulations $[13,16]$ suggested that the shear band regions tend to be disordered and exhibit liquidlike behavior. A phase field approach [65] would be useful to account for this effect and also to describe melting due to dislocation proliferation.

(x) In this work we have not constructed macroscopic laws such as constitutive equations. To this end we need deeper understanding of dislocation dynamics.

\section{ACKNOWLEDGMENTS}

This work was supported by Grants in Aid for Scientific Research from the Ministry of Education, Science and Culture, Japan. The author thanks R. Yamamoto for valuable discussions. Thanks are also due to A. S. Argon for sending his papers and informative correspondence.

\section{APPENDIX}

Here we examine the linearized equations (2.16) and (2.17) around a homogeneously strained state with $\left\langle e_{1}\right\rangle$ $=0,\left\langle e_{3}\right\rangle=\gamma$, and $\left\langle e_{2}\right\rangle=\epsilon$. We neglect the random stress and assume that all the deviations depend on space and time as $\exp (i \boldsymbol{k} \cdot \boldsymbol{r}+i \omega t)$. Then the deviation of the displacement vector $\left(\delta u_{x}, \delta u_{y}\right)$ obeys

$$
\begin{aligned}
& \Omega \delta u_{x}=C_{x x} \delta u_{x}+C_{x y} \delta u_{y}, \\
& \Omega \delta u_{y}=C_{x y} \delta u_{x}+C_{y y} \delta u_{y},
\end{aligned}
$$

where

$$
\Omega=\rho \omega^{2} / k^{2}-i \eta_{0} \omega
$$

The frequency $\omega$ is expressed in terms of $\Omega$ at small $k$ as

$$
\omega= \pm(\Omega / \rho)^{1 / 2} k+i\left(\eta_{0} / 2 \rho\right) k^{2}+O\left(k^{3}\right) .
$$

The first term is the oscillation frequency for $\Omega>0$ and the second term viscous damping. The coefficients $C_{\alpha \beta}$ are expressed in terms of the coefficients $\Phi_{\alpha \beta}$ in Eq. (2.7) as 


$$
\begin{gathered}
C_{x x}=\left(K_{0}+\Phi_{22}\right) n_{x}^{2}+2 \Phi_{23} n_{x} n_{y}+\Phi_{33} n_{y}^{2}, \\
C_{y y}=\left(K_{0}+\Phi_{22}\right) n_{y}^{2}-2 \Phi_{23} n_{x} n_{y}+\Phi_{33} n_{x}^{2}, \\
C_{x y}=\left(K_{0}-\Phi_{22}+\Phi_{33}\right) n_{x} n_{y}+\Phi_{23}\left(n_{x}^{2}-n_{y}^{2}\right) .
\end{gathered}
$$

Here $\boldsymbol{n}=k^{-1} \boldsymbol{k}$ is the unit vector representing the direction of the wave vector $\boldsymbol{k}$. From Eq. (A1) it follows the relation

$$
\left(\Omega-C_{x x}\right)\left(\Omega-C_{y y}\right)=C_{x y}^{2} .
$$

Let the angle of $\boldsymbol{n}$ be $\varphi+\pi / 2$ with respect to the $x$ axis; then,

$$
n_{x}=-\sin \varphi, \quad n_{y}=\cos \varphi .
$$

After some calculations Eq. (A5) is solved to give

$$
\begin{aligned}
\Omega & =\frac{1}{2}\left(K_{0}+\Phi_{22}+\Phi_{33}\right) \\
& \pm \sqrt{\frac{1}{4} K_{0}^{2}+A^{2}+B^{2}+K_{0}(A \cos 4 \varphi+B \sin 4 \varphi)}
\end{aligned}
$$

where $A=\left(\Phi_{22}-\Phi_{33}\right) / 2$ and $B=\Phi_{23}$. As a function of $\varphi$ the slowest mode is obtained if the combination $A \cos 4 \varphi$ $+B \sin 4 \varphi$ takes the maximum $\left(A^{2}+B^{2}\right)^{1 / 2}$. The corresponding minimum of $\Omega$ is given by

$$
\Omega_{\min }=\frac{1}{2}\left(\Phi_{22}+\Phi_{33}\right)-\sqrt{A^{2}+B^{2}} .
$$

Notice that $\Omega_{\min }$ is the smaller of the two eigenvalues of the matrix $\left\{\Phi_{\alpha \beta}\right\}$. We may draw two conclusions.

(i) As the instability point is approached, $\Omega_{\min }$ tends to zero with softening of the sound speed $\left(\Omega_{\min } / \rho\right)^{1 / 2}$ of the corresponding acoustic mode.

(ii) If the approximate expression (2.8) is used, we have $A=2 G^{\prime \prime}\left(\epsilon^{2}-\gamma^{2}\right)$ and $B=4 G^{\prime \prime} \epsilon \gamma$ so that

$$
A \cos 4 \varphi+B \sin 4 \varphi=C \cos (4 \varphi+2 \alpha),
$$

where $C=2\left|G^{\prime \prime}\right|\left(\epsilon^{2}+\gamma^{2}\right)$ and $\alpha$ is defined by Eq. (3.15). Thus the minimum condition for $\Omega$ yielding Eq. (A8) is given by $4 \varphi+2 \alpha=2 n \pi$ and is equivalent to Eq. (3.14). That is, the slowest mode, which undergoes softening at the instability point, has a wave vector perpendicular to the favorable slip orientations given by Eq. (3.14). This is the case even far below the instability point. In addition, for the slowest mode, Eq. (A1) gives

$$
\delta u_{y} / \delta u_{x}=\left(\Omega_{\min }-C_{x x}\right) / C_{x y}=\cot \varphi,
$$

if $\alpha$ is eliminated using Eq. (3.14). Thus the deviation $\delta \boldsymbol{u}$ is perpendicular to the wave vector $\boldsymbol{k}$ or the slowest mode is a transverse sound.
[1] J. Friedel, Dislocations (Pergamon, New York, 1954).

[2] F.R.N. Nabarro, Theory of Dislocations (Clarendon, Oxford, 1967).

[3] B. Devincre and L.P. Kubin, Mater. Sci. Eng., A 234, 8 (1997).

[4] V. Bulatov, F.F. Abraham, L. Kubin, B. Devincre, and S. Yip, Nature (London) 391, 669 (1998).

[5] K.W. Schwarz, J. Appl. Phys. 85, 108 (1999).

[6] A. Artemev, Y. Jin, and A.G. Khachaturyan, Acta Mater. 77, 1165 (2001).

[7] J.J. Gilman, J. Appl. Phys. 46, 1625 (1975).

[8] F. Spaepen, Acta Metall. 25, 407 (1977).

[9] A.S. Argon, Acta Metall. 27, 47 (1979).

[10] F. Spaepen and A. Taub, in Amorphous Metallic Alloys, edited by F. Luborsky (Butterworths, London, 1983), p. 231.

[11] H. Kimura and T. Matsumoto, Amorphous Metallic Alloys, Ref. [10], p. 187.

[12] F. Spaepen, in Physics of Defects, edited by R. Balian, M. Kléman, and J-P. Poirier, 1981 Les Houches Lectures (NorthHolland, Amsterdam, 1981), p. 133.

[13] A.S. Argon, in Material Sciences and Technology, edited by R.W. Cohen, P. Haasen, and E.J. Kramer (VCH, Weinheim, 1993), Vol. 6.

[14] Jing Li, F. Spaepen, and T.C. Hufnagel, Philos. Mag. A 82, 2623 (2002).

[15] In this work we do not consider fracture or brittle behavior. Following Argon et al. [16,18], we use the term "shear band" widely to represent quasilinear (in 2D) [or quasiplanar (in 3D)] objects where shear strains are localized. The narrowest ones are slips composed of dislocation pairs.

[16] D. Deng, A.S. Argon, and S. Yip, Philos. Trans. R. Soc. London, Ser. A 329, 613 (1989).
[17] X.Y. Fu, D.A. Rigney, and M.L. Falk, J. Non-Cryst. Solids 317, 206 (2003).

[18] V.V. Bulatov and A.S. Argon, Modell. Simul. Mater. Sci. Eng. 2, 167 (1994).

[19] K. Maeda and S. Takeuchi, Phys. Status Solidi A 49, 685 (1978); S. Kobayashi, K.K. Maeda, and S. Takeuchi, Acta Metall. 28, 1641 (1980); K. Maeda and S. Takeuchi, Philos. Mag. A 44, 643 (1981).

[20] T. Muranaka and Y. Hiwatari, Phys. Rev. E 51, R2735 (1995).

[21] D.N. Perera and P. Harrowell, Phys. Rev. E 54, 1652 (1996).

[22] R. Yamamoto and A. Onuki, J. Phys. Soc. Jpn. 66, 2545 (1997).

[23] W. Kob, C. Donati, S.J. Plimpton, P.H. Poole, and S.C. Glotzer, Phys. Rev. Lett. 79, 2827 (1997).

[24] R. Yamamoto and A. Onuki, Europhys. Lett. 40, 61 (1997); Phys. Rev. E 58, 3515 (1998).

[25] R. Yamamoto and A. Onuki, J. Phys.: Condens. Matter 12, $6323(2000)$.

[26] J.H. Simmons, R.K. Mohr, and C.J. Montrose, J. Appl. Phys. 53, 4075 (1982); J.H. Simmons, R. Ochoa, K.D. Simmons, and J.J. Mills, J. Non-Cryst. Solids 105, 313 (1988).

[27] H. Kato, Y. Kawamura, A. Inoue, and H.S. Chen, Appl. Phys. Lett. 73, 3665 (1998).

[28] R.R. Balokhonov, P.V. Makarov, V.A. Romanova, and I.Y. Smolin, Comput. Mater. Sci. 16, 355 (1999).

[29] B. Crist, in The Physics of Glassy Polymers, edited by R.N. Haward and R.J. Young (Chapman and Hall, London, 1997), p. 155.

[30] M.C. Boyce and R.N. Haward, in The Physics of Glassy Polymers, Ref. [29], p. 213. 
[31] P.H. Mott, A.S. Argon, and U.W. Suter, Philos. Mag. A 67, 931 (1993); A.S. Aragon, V.V. Bulatov, P.H. Mott, and U.W. Suter, J. Rheol. 39, 377 (1995).

[32] R. Yamamoto and A. Onuki, J. Chem. Phys. 117, 2359 (2002).

[33] Jamming and Rheology, edited by A. Liu and S.R. Nagel (Taylor \& Francis, London, 2001). Here a number of related papers can be found.

[34] T. Okuzono and K. Kawasaki, Phys. Rev. E 51, 1246 (1995).

[35] D.J. Durian, Phys. Rev. E 55, 1739 (1997); S.A. Langer and A.J. Liu, J. Phys. Chem. B 101, 8667 (1997).

[36] J. Desrues, J. Lanier, and P. Stutz, Eng. Fract. Mech. 21, 909 (1985).

[37] J. Desrues and R. Chambon, Int. J. Solids Struct. 39, 3757 (2002).

[38] C.T. Veje, D.W. Howell, and R.P. Behringer, Phys. Rev. E 59, 739 (1999).

[39] A. Onuki, J. Phys.: Condens. Matter 15, S891 (2003). A simpler 1D version similar to that in Ref. [46] follows if we set $u_{y}=0$ and assume homogeneity along the $x$ axis. In this version, the stress in plastic flow behaves as $\dot{\gamma}^{0.3}$, which is incorrectly written as $\dot{\gamma}^{0.7}$ in Ref. [39].

[40] Typical applied stress is tensile, compressive, or shear. In experiments uniaxial deformation is more usual than shear deformation.

[41] M.L. Falk and J.S. Langer, Phys. Rev. E 57, 7192 (1998).

[42] A.S. Argon and H.Y. Kuo, Mater. Sci. Eng. 39, 101 (1979).

[43] M. Peach and J.S. Koehler, Phys. Rev. 80, 436 (1950).

[44] R. Peierls, Proc. Phys. Soc. 52, 34 (1940).

[45] F.R.N. Nabarro, Proc. Phys. Soc. 59, 256 (1947).

[46] M. Doi, J.L. Harden, and T. Ohta, Macromolecules 26, 4935 (1993)

[47] P.M. Chaikin and T.C. Lubensky, Principles of Condensed Matter Physics (Cambridge University Press, Cambridge, 1995), pp. 601-620.

[48] A. Onuki, Phase Transition Dynamics (Cambridge University Press, Cambridge, 2002).

[49] If Eq. (2.12) is assumed, the elastic energy on the basis of Eq. (2.5) becomes a functional of $\boldsymbol{u}$ only. Then the resultant stress tensor following from Eq. (2.13) contains a small antisymmetric part or $\sigma_{x y}-\sigma_{y x} \neq 0$ on the order of $e^{6}$ from expansion (2.6). This suggests room of improvement for our nonlinear strain theory.

[50] L.D. Landau and E.M. Lifshitz, Theory of Elasticity (Pergamon, New York, 1973).

[51] In 2D the viscous stress tensor is written as $\eta_{0}\left(\nabla_{i} v_{j}+\nabla_{j} v_{i}\right.$ $\left.-\delta_{i j} \boldsymbol{\nabla} \cdot \boldsymbol{v}\right)+\zeta_{0} \delta_{i j} \nabla \cdot \boldsymbol{v}$, where $\eta_{0}$ and $\zeta_{0}$ are the shear and bulk viscosities, respectively [50].

[52] If $\rho$ is included as a dynamic variable, $\rho$ obeys the continuity equation $\partial \rho / \partial t=-\boldsymbol{\nabla} \cdot(\rho \boldsymbol{v})$ and the right hand side of Eq. (2.17) should be replaced by $\rho[\partial / \partial t+\boldsymbol{v} \cdot \boldsymbol{\nabla}] \boldsymbol{v}$. Then the selfconsistency relation (2.20) is kept to be valid. For large strains of order 1 this generalization is needed.

[53] This slip energy may be calculated in the linear elasticity theory [50]. If Eq. (3.3) is substituted into the elastic energy expression, a logarithmic space integral $\left(\propto \int d r r^{-1}\right)$ appears in the range $a \leq r \leq \ell$. The lower bound is provided by nonlinear elasticity theory. This slip energy also follows from Eq. (3.11).

[54] In 2D, the glide direction is along the Burgers vector $\boldsymbol{b}$, and the climb direction is perpendicular to $\boldsymbol{b}[1,2]$.

[55] J.W. Rudnicki and J.R. Rice, J. Mech. Phys. Solids 23, 371 (1975).

[56] J.S. Langer, Phys. Rev. E 64, 011504 (2001).

[57] H. Ikeda, Y. Qi, T. Çagin, K. Samwer, W.L. Johnson, and W.A. Goddard III, Phys. Rev. Lett. 82, 2900 (1999).

[58] The Peierls potential energy depends on the ratio of the atomic spacing across the glide plane and the magnitude of the Burgers vector $[1,2,44,45]$. As a result, the critical stress causing dislocation motion (Peierls stress) can strongly depend on the orientation of the glide plane with respect to the crystal axes.

[59] M. Utz, P.G. Debenedetti, and F.H. Stillinger, Phys. Rev. Lett. 84, 1471 (2000).

[60] D.J. Lacks, Phys. Rev. Lett. 87, 225502 (2001).

[61] L. Angelani, G. Ruocco, F. Sciortino, P. Tartaglia, and F. Zamponi, Phys. Rev. E 66, 061505 (2002).

[62] W. Kob, F. Sciortino, and P. Tartaglia, Europhys. Lett. 49, 590 (2000).

[63] A.S. Argon (private communication).

[64] D. Walgraef and E.C. Aifantis, J. Appl. Phys. 58, 688 (1985); P. Hähner, K. Bay, and M. Zaiser, Phys. Rev. Lett. 81, 2470 (1998).

[65] R. Kobayashi, Physica D 63, 410 (1993). 